\title{
Conjugated Thiophene-Fused Isatin Dyes through Intramolecular Direct Arylation
}

Andrea Nitti, ${ }^{a}$ Marco Signorile, ${ }^{a}$ Massimo Boiocchi, ${ }^{b}$ Gabriele Bianchi, ${ }^{c}$ Riccardo Po ${ }^{c}$ and Dario Pasini ${ }^{\text {a,d }}$
a) Department of Chemistry, University of Pavia, Viale Taramelli, 12 - 27100 Pavia -Italy.
b) Centro Grandi Strumenti, University of Pavia, Via Bassi 21, 27100 Pavia, Italy.
c) Research Center for Renewable Energies and Environment, Istituto Donegani, Eni Spa, Via Fauser 4, 28100 Novara, Italy.
d) INSTM Research Unit, University of Pavia, Viale Taramelli, 12 - 27100 Pavia -Italy. Email: dario.pasini@unipv.it

\section{Table of Contents:}

Additional Experiments 


\section{Additional Experiments}

Acylation of isatin 1 with 3-thiophene carbonyl chloride, under the conditions reported in Table S1 have led to the isolation of starting isatin after work up.

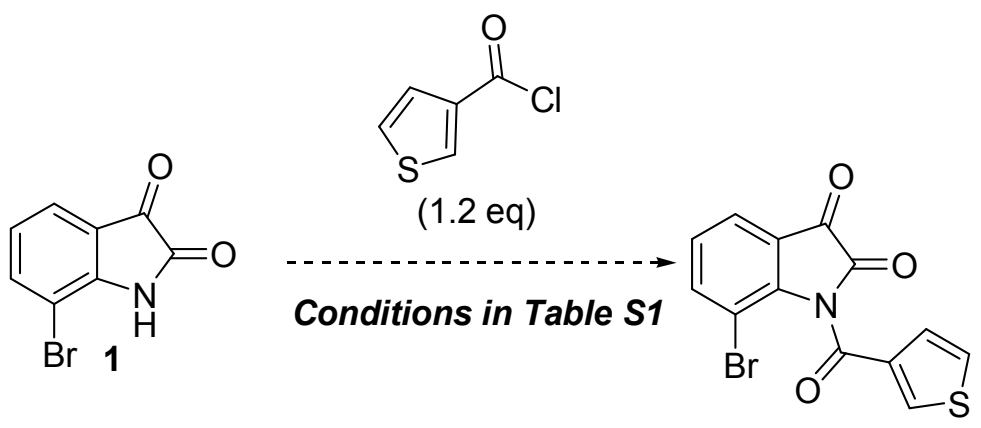

Table S1. Conditions for the attempted acylation of isatin $\mathbf{1 .}$

\begin{tabular}{clc}
\hline Entry & \multicolumn{1}{c}{ Method } & Conv [\%] \\
\hline 1 & NaH (1.5eq), THF dry, $0^{\circ}$ C-rt. & 0 \\
2 & DMAP (0.2eq), triethylamine (1.1eq), THF dry. & 0 \\
3 & DMAP (0.2eq), triethylamine (1.1 eq), DCM dry. & 0 \\
4 & Pyridine (2.1 eq), DCM dry. & 0 \\
\hline
\end{tabular}


UV-Vis and CV spectra of compounds 5 and 23

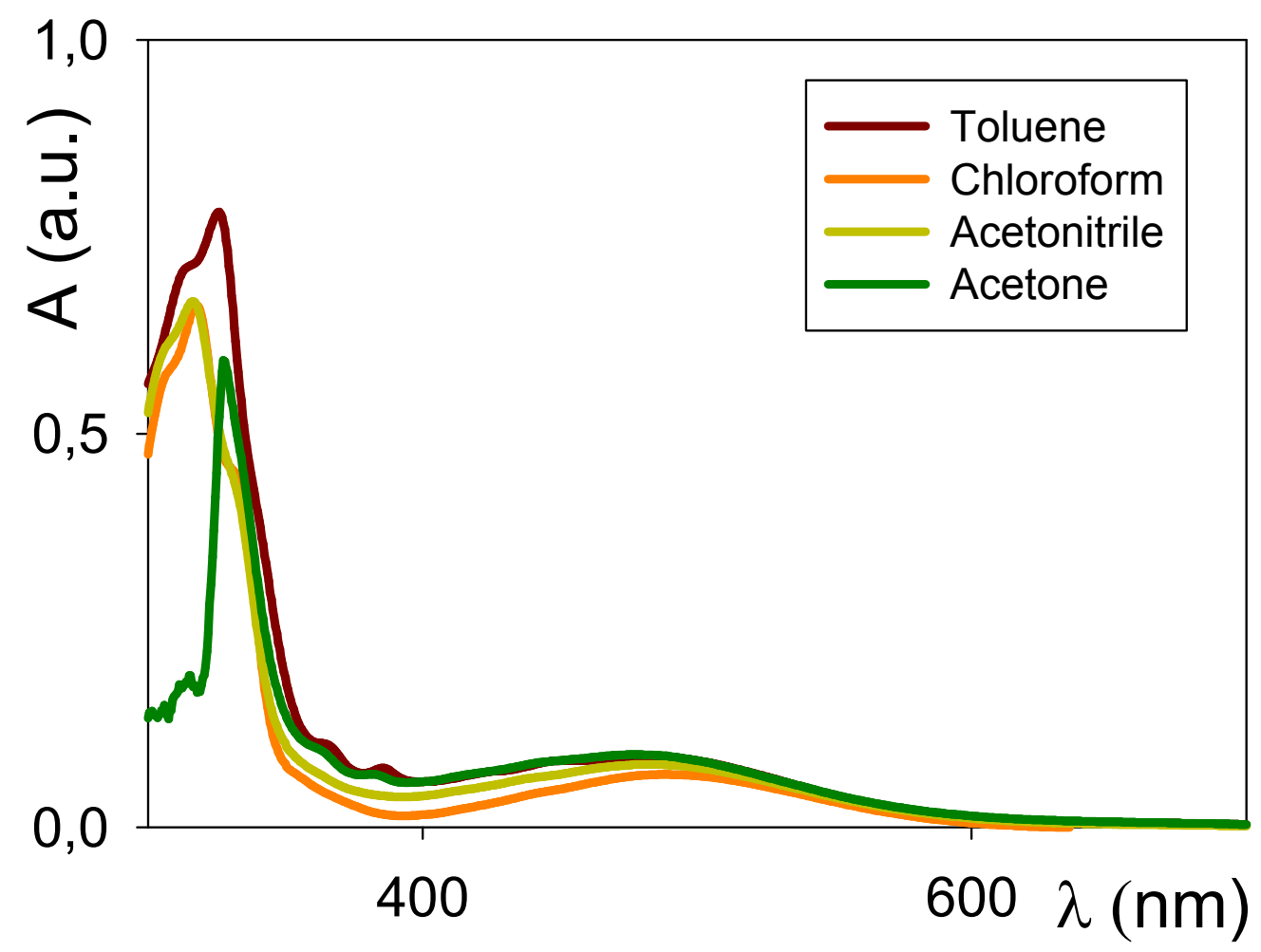

Figure S1. UV/Vis spectra of compounds $5\left(\right.$ ca. $\left.5 \times 10^{-6} \mathrm{M}\right)$ in different solvents. 

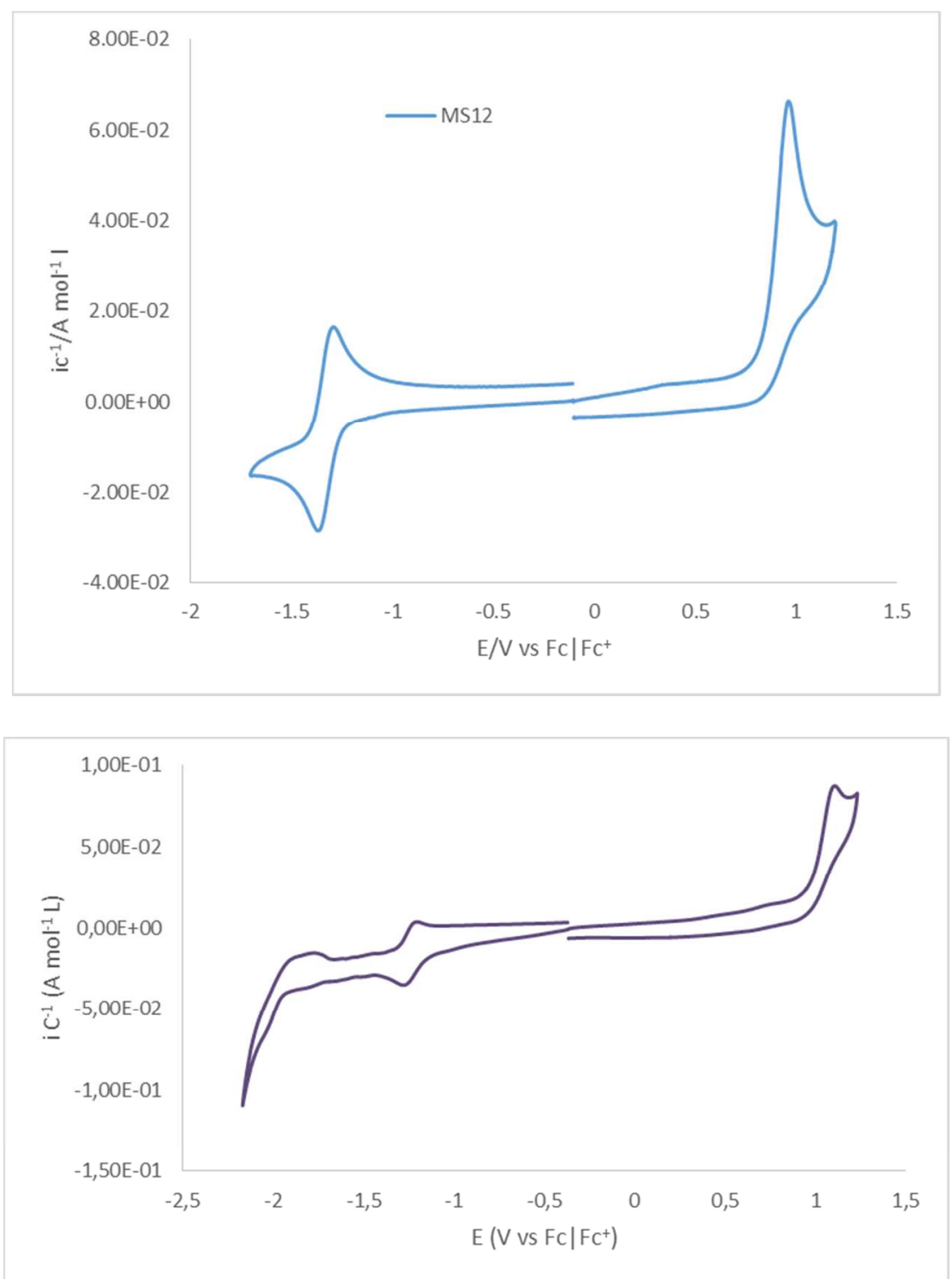

Figure S2. Cyclic Voltammetry of compound 5 (top) and 23 (bottom). For conditions, see General Experimental Section in the manuscript. 


\section{Additional X-Ray Figures}
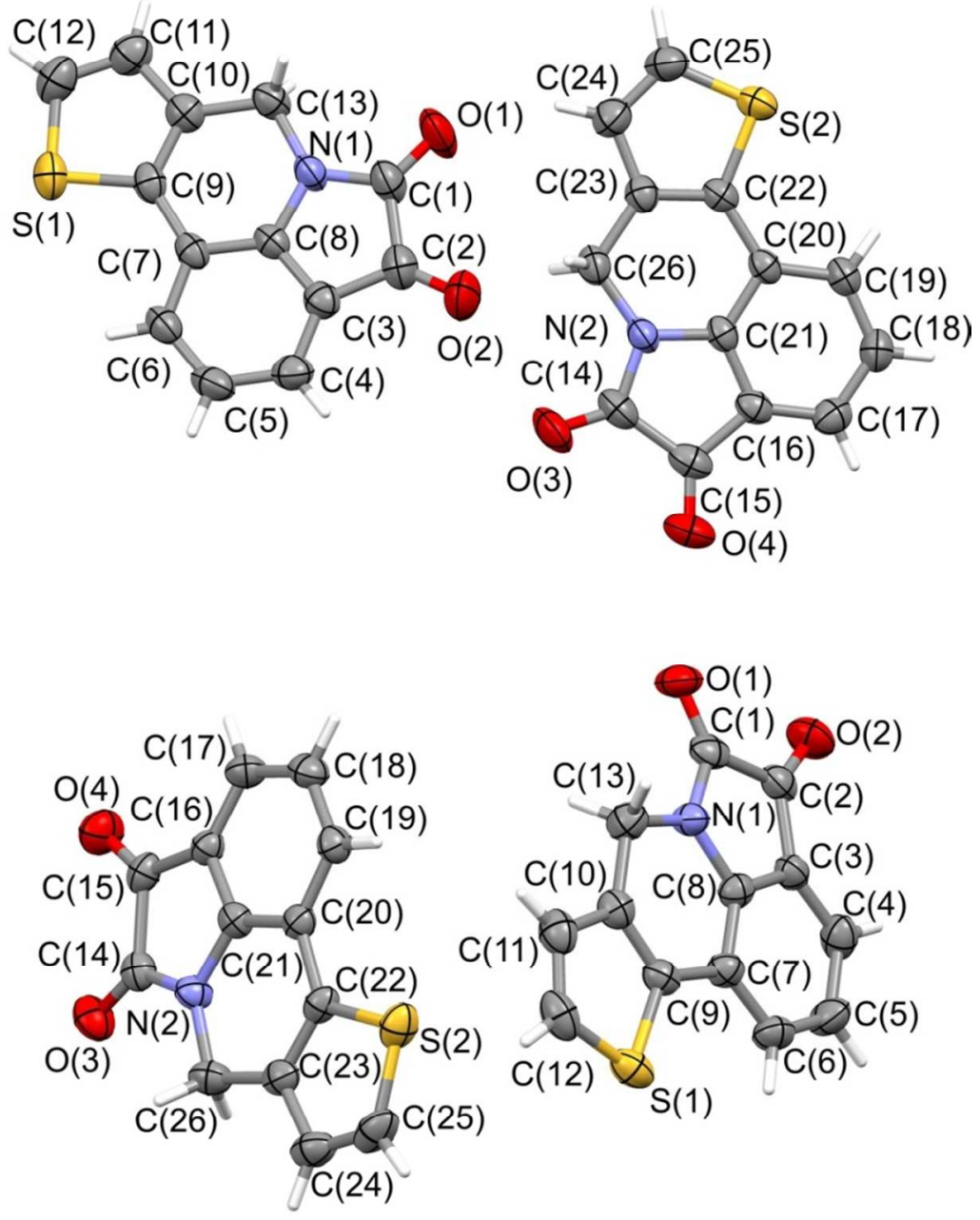

Figure S3. Plots showing thermal ellipsoids for the two independent molecules in the 5_1 crystal (above) and in the 5_2 crystal (below). Ellipsoids are drawn at the 50\% probability level. Atom names are reported only for non-hydrogen atoms 


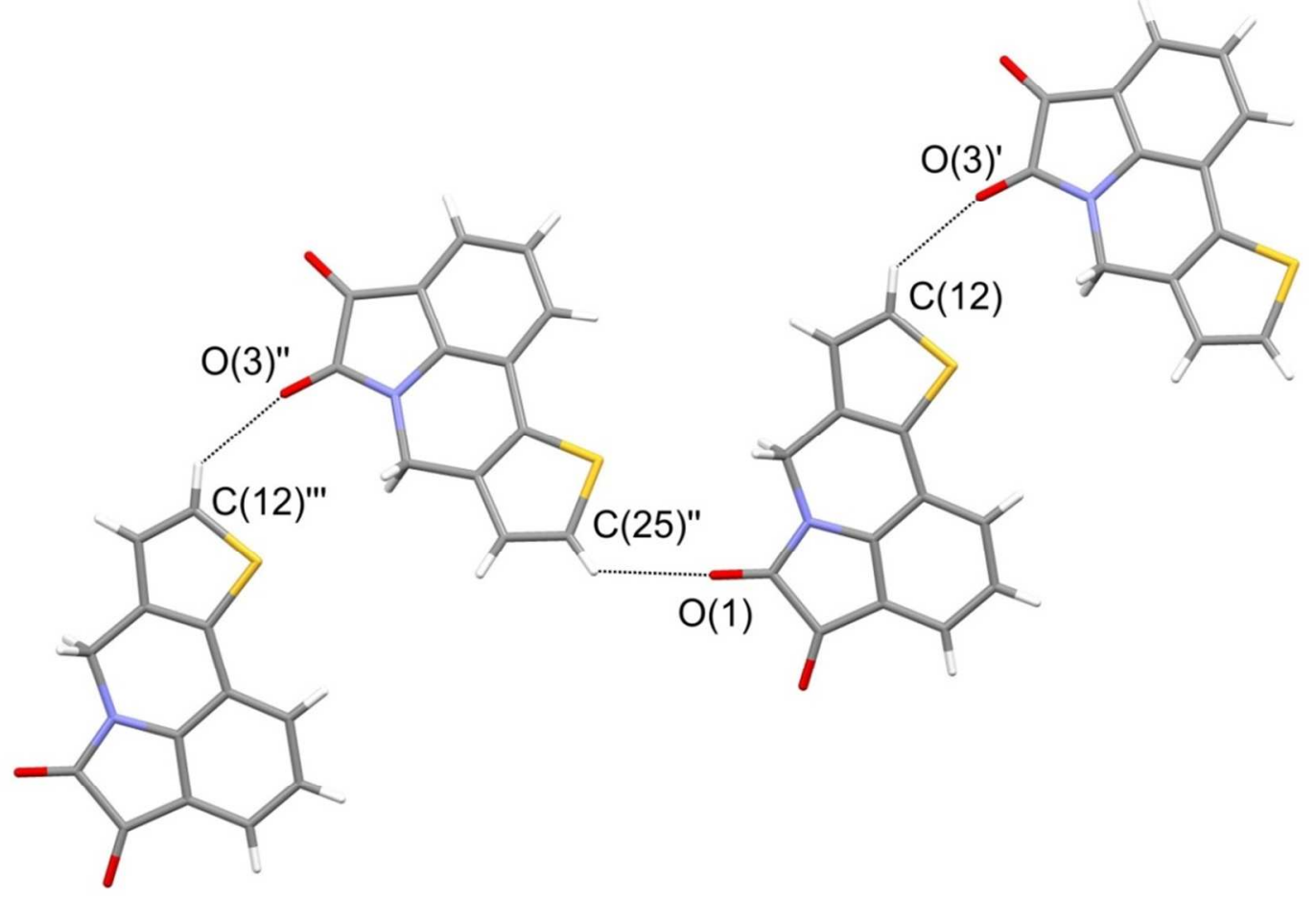

Figure S4. The C-H $\cdots \mathrm{O}$ hydrogen bond motif occurring between couples of independent molecules in the 5_1 crystal. Complete H-bonds are shown only for the two central molecules. Features for the independent $\mathrm{C}-\mathrm{H} \cdots \mathrm{O}$ interactions are: $\mathrm{C}(12) \cdots \mathrm{O}(3)^{\prime} 3.24(1) \AA, \mathrm{H}(12) \cdots \mathrm{O}(3)^{\prime} 2.51(1) \AA, \mathrm{C}(12)-$ $\mathrm{H}(12) \cdots \mathrm{O}(3)^{\prime} 135.4(2)^{\circ} ; \quad \mathrm{C}(25)^{\prime \prime} \cdots \mathrm{O}(1) 3.22(1) \AA, \mathrm{H}(25)^{\prime} \cdots \mathrm{O}(1) 2.57(1) \AA, \mathrm{C}(25)^{\prime \prime}-\mathrm{H}(25)^{\prime \prime} \cdots \mathrm{O}(1)$ $127.8(2)^{\circ}$. Symmetry code: (') $=1-x, 1-y, 2-z ;(")=2-x, 1-y, 1-z ;(")=1+x, y,-1+z$. 


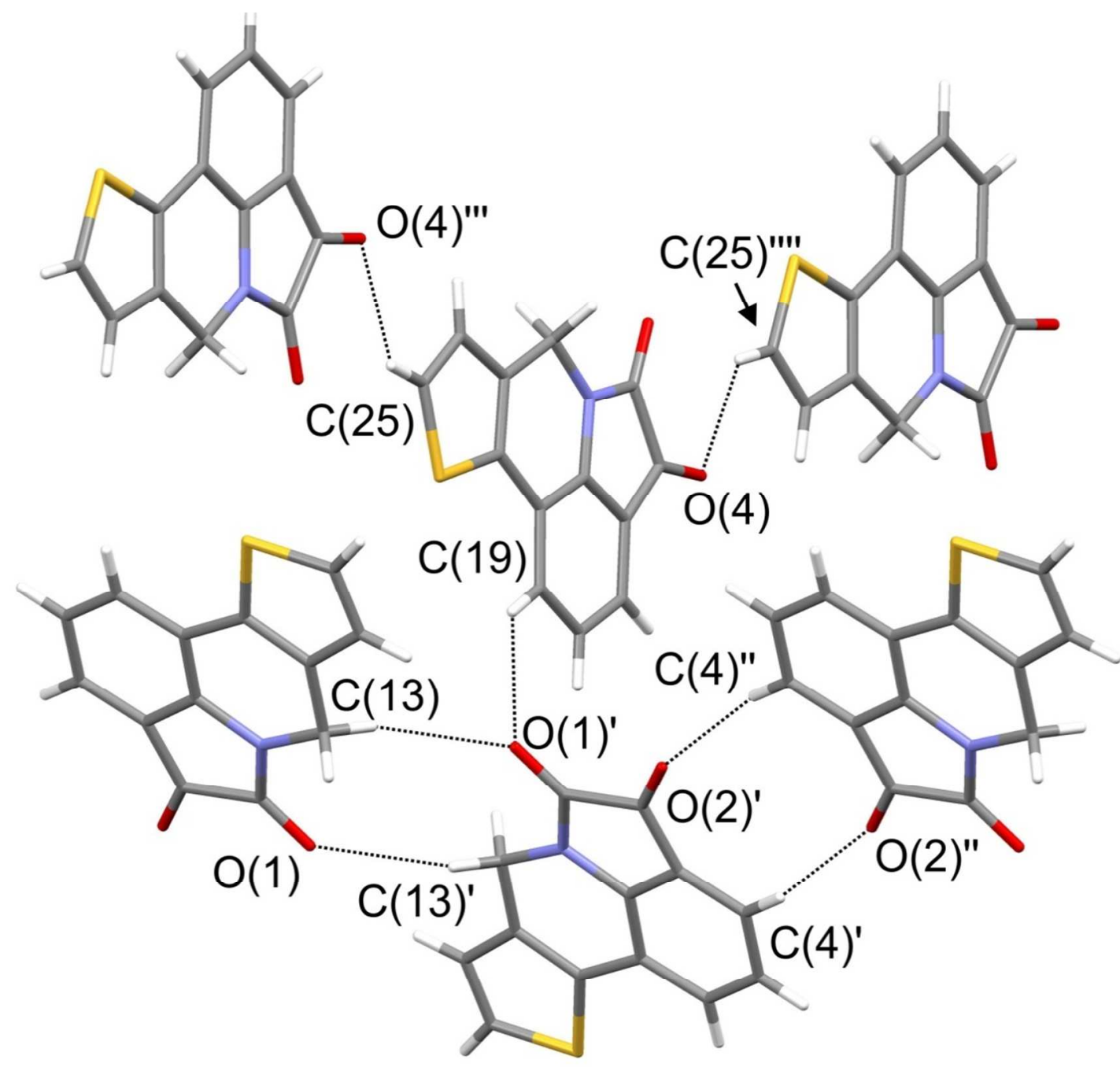

Figure S5. The $\mathrm{C}-\mathrm{H} \cdots \mathrm{O}$ hydrogen bond motif in the $\mathbf{5} \mathbf{2}$ crystal. H-bonded chains made by symmetrically equivalent molecules are interconnected by additional $\mathrm{C}-\mathrm{H} \cdots \mathrm{O}$ interactions between couples of independent molecules. Complete H-bonds are shown only for the two molecules in the middle. Features for the independent $\mathrm{C}-\mathrm{H} \cdots \mathrm{O}$ interactions are: $\mathrm{C}(4)^{\prime} \cdots \mathrm{O}(2)^{\prime \prime} 3.46(1) \AA$, $\mathrm{H}(4)^{\prime} \cdots \mathrm{O}(2)^{\prime \prime}$ 2.55(1) $\AA, \mathrm{C}(4)^{\prime}-\mathrm{H}(4)^{\prime} \cdots \mathrm{O}(2)^{\prime \prime} 166.5(2)^{\circ} ; \mathrm{C}(13) \cdots \mathrm{O}(1)^{\prime} 3.39(1) \AA, \mathrm{H}(13) \cdots \mathrm{O}(1)^{\prime} 2.54(1) \AA ⿻$ $\mathrm{H}(13) \cdots \mathrm{O}(1)^{\prime} 147.1(2)^{\circ} ; \mathrm{C}(19) \cdots \mathrm{O}(1)^{\prime} 3.27(1) \AA \AA \mathrm{H}(19) \cdots \mathrm{O}(1)^{\prime} 2.56(1) \AA, \mathrm{C}(19)-\mathrm{H}(19)^{\cdots} \mathrm{O}(1)^{\prime}$

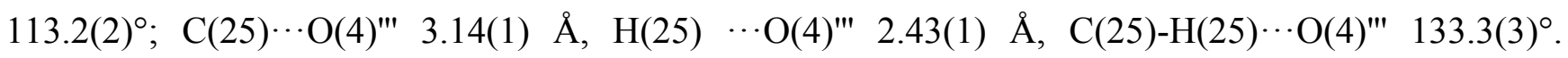
Symmetry code: (') $=1-x,-y, 1-z ;(")=-1+x, y, 1+z ;(" ')=-3 / 2+x, 1 / 2-y,-1 / 2+z ;(" ')=3 / 2+x, 1 / 2-y, 1 / 2+z$; 

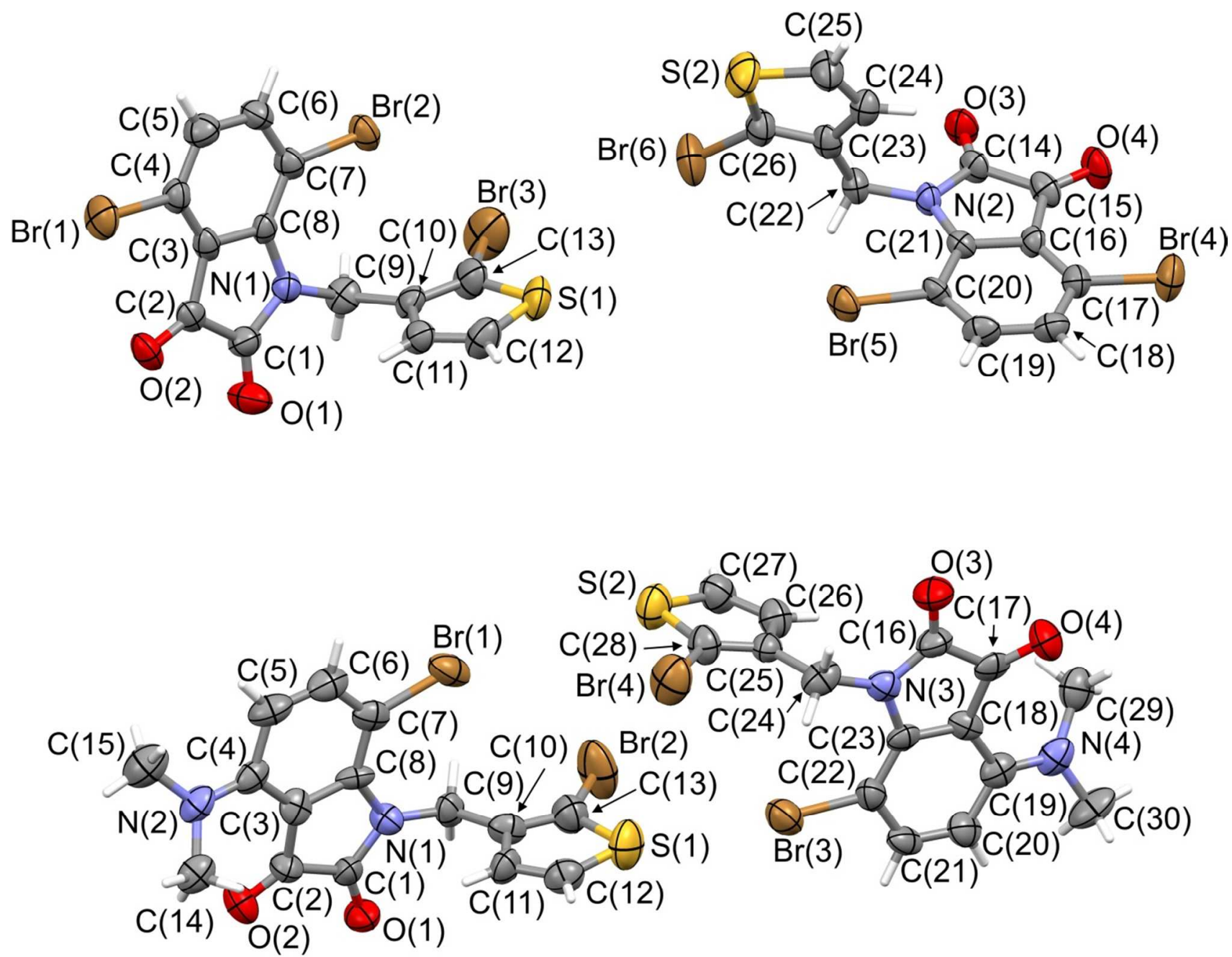

Figure S6. Plots showing thermal ellipsoids for the two similar but not equivalent molecules occurring in the crystals of $\mathbf{1 5}$ (above) and $\mathbf{1 7}$ (below) compounds (ellipsoids are drawn at the 50\% probability level). For the crystal of $\mathbf{1 5}$, the additional dichloromethane solvent molecule is not shown for clarity. 
Table S2. Bond distances $(\AA)$ for the ring structures of the four crytrallographically-independent molecules of compound 5 (two polymorphs 5_1 and 5_2). The corresponding distances for the two independent molecules of each crystal polymorph are reported on the same line.

\begin{tabular}{|c|c|c|c|c|c|c|c|}
\hline \multicolumn{4}{|c|}{ 5_1 } & \multicolumn{4}{|c|}{ 5_2 } \\
\hline \multicolumn{4}{|c|}{ isatin } & \multicolumn{4}{|c|}{ isatin } \\
\hline $\mathrm{C}(1)-\mathrm{N}(1)$ & $1.361(3)$ & $\mathrm{C}(14)-\mathrm{N}(2)$ & $1.363(3)$ & $\mathrm{C}(1)-\mathrm{N}(1)$ & $1.362(4)$ & $\mathrm{C}(14)-\mathrm{N}(2)$ & $1.366(4)$ \\
\hline $\mathrm{C}(1)-\mathrm{O}(1)$ & $1.208(3)$ & $\mathrm{C}(14)-\mathrm{O}(3)$ & $1.212(2)$ & $\mathrm{C}(1)-\mathrm{O}(1)$ & $1.210(4)$ & $\mathrm{C}(14)-\mathrm{O}(3)$ & $1.214(4)$ \\
\hline $\mathrm{C}(2)-\mathrm{O}(2)$ & $1.208(3)$ & $\mathrm{C}(15)-\mathrm{O}(4)$ & $1.208(2)$ & $\mathrm{C}(2)-\mathrm{O}(2)$ & $1.206(4)$ & $\mathrm{C}(15)-\mathrm{O}(4)$ & $1.210(4)$ \\
\hline $\mathrm{C}(1)-\mathrm{C}(2)$ & $1.571(3)$ & $C(14)-C(15)$ & $1.553(3)$ & $\mathrm{C}(1)-\mathrm{C}(2)$ & $1.564(5)$ & $C(14)-C(15)$ & $1.566(5)$ \\
\hline $\mathrm{C}(2)-\mathrm{C}(3)$ & $1.462(3)$ & $C(15)-C(16)$ & $1.472(3)$ & $C(2)-C(3)$ & $1.467(5)$ & $C(15)-C(16)$ & $1.455(5)$ \\
\hline$C(3)-C(4)$ & & $C(16$ & $1.380(3)$ & $C(3)-C(4)$ & $1.384(5)$ & $C(16)-C(17)$ & $1.393(5)$ \\
\hline$C(3)$ & & $C(16$ & 1.386 & $C(3$ & & $\mathrm{C}(1 \mathrm{C}$ & \\
\hline$C(4)-C(5)$ & $1.387(3)$ & $\mathrm{C}(17)-\mathrm{C}(18)$ & $1.386(3)$ & $C(4)-C(5)$ & $1.376(5)$ & $\mathrm{C}(17)-\mathrm{C}(18)$ & $1.376(5)$ \\
\hline$C(5)-C(6)$ & $1.384(3)$ & $\mathrm{C}(18)-\mathrm{C}(19)$ & $1.392(3)$ & $C(5)-C(6)$ & $1.393(5)$ & $C(18)-C(19)$ & $1.384(5)$ \\
\hline$C(6$ & & & & $C(6$ & $1.389(5)$ & $C(19)$ & $1.389(5)$ \\
\hline$C(7)$ & $1.385(3)$ & $C(20$ & $1.385(2)$ & $C(7)-$ & $1.378(5)$ & $\mathrm{C}(20)$ & $1.377(4)$ \\
\hline \multicolumn{4}{|c|}{ thiophene } & \multicolumn{4}{|c|}{ thiophene } \\
\hline $\mathrm{C}(9)-\mathrm{S}(1)$ & $1.728(3)$ & $\mathrm{C}(22)-\mathrm{S}(2)$ & $1.722(2)$ & $\mathrm{C}(9)-\mathrm{S}(1)$ & $1.714(4)$ & $\mathrm{C}(22)-\mathrm{S}(2)$ & $1.717(3)$ \\
\hline $\mathrm{C}(1)$ & & & $1(2)$ & $\mathrm{C}(1$ & $1.702(4)$ & & \\
\hline $\mathrm{C}(9)-\mathrm{C}(10)$ & $1.374(3)$ & $\mathrm{C}(22)$ & $1.373(3)$ & $C(9)-$ & $1.377(5)$ & $C(22)-C(23)$ & $1.376(5)$ \\
\hline$C(11)-C(12)$ & $1.346(3)$ & $C(24)-C(25)$ & $1.347(3)$ & $\mathrm{C}(11)-\mathrm{C}(12)$ & $1.360(5)$ & $C(24)-C(25)$ & $1.340(5)$ \\
\hline $\mathrm{C}(10)-\mathrm{C}(11)$ & $\begin{array}{l}1.421(3) \\
\text { fused }\end{array}$ & $\begin{array}{l}\mathrm{C}(23)-\mathrm{C}(24) \\
\text { ring }\end{array}$ & $1.419(3)$ & \multicolumn{3}{|c|}{ fused ring } & $1.420(5)$ \\
\hline \multicolumn{4}{|c|}{ fused ring } & $C(7)$ & $1.459(5)$ & $C(20)-C(22)$ & $1.455(4)$ \\
\hline$C(10)-$ & $1.501(3)$ & $C(23)$ & $1.504(3)$ & $\mathrm{C}(10)_{-}$ & $1.495(5)$ & $C(23)-C(26)$ & $1.509(5)$ \\
\hline $\mathrm{C}(13)-\mathrm{N}(1)$ & $1.464(3)$ & $\mathrm{C}(26)-\mathrm{N}(2)$ & $1.466(3)$ & $\mathrm{C}(13)-\mathrm{N}(1)$ & $1.459(4)$ & $\mathrm{C}(26)-\mathrm{N}(2)$ & $1.457(4)$ \\
\hline
\end{tabular}


Table S3. Crystal data for investigated crystals.

\begin{tabular}{|c|c|c|c|c|}
\hline & $5 \_1$ & $5 \_2$ & 15 & 17 \\
\hline Formula & $\mathrm{C}_{26} \mathrm{H}_{14} \mathrm{~N}_{2} \mathrm{O}_{4} \mathrm{~S}_{2}$ & $\mathrm{C}_{26} \mathrm{H}_{14} \mathrm{~N}_{2} \mathrm{O}_{4} \mathrm{~S}_{2}$ & $\mathrm{C}_{27} \mathrm{H}_{14} \mathrm{Br}_{6} \mathrm{Cl}_{2} \mathrm{~N}_{2} \mathrm{O}_{4} \mathrm{~S}_{2}$ & $\mathrm{C}_{30} \mathrm{H}_{24} \mathrm{Br}_{4} \mathrm{~N}_{4} \mathrm{O}_{4} \mathrm{~S}_{2}$ \\
\hline$M$ & 482.52 & 482.52 & 1044.82 & 888.26 \\
\hline Crystal system & triclinic & monoclinic & triclinic & monoclinic \\
\hline Space group & $P-1$ (no. 2$)$ & $P 2_{1} / n$ (no. 14$)$ & $P-1$ (no. 2$)$ & $P 2_{1} / c$ (no. 14$)$ \\
\hline$a[\AA]$ & $7.331(2)$ & $5.3775(6)$ & $8.205(2)$ & $23.534(4)$ \\
\hline$b[\AA]$ & $10.1068(15)$ & $31.052(5)$ & $14.884(4)$ & $8.2908(12)$ \\
\hline$c[\AA]$ & $14.572(2)$ & $12.3237(13)$ & $14.978(4)$ & $16.250(6)$ \\
\hline$\alpha\left[^{\circ}\right]$ & $84.948(15)$ & 90 & $62.98(2)$ & 90 \\
\hline$\beta\left[^{\circ}\right]$ & $83.20(3)$ & $90.673(11)$ & $86.64(3)$ & $96.99(2)$ \\
\hline$\gamma\left[^{\circ}\right]$ & $79.94(14)$ & 90 & $86.32(3)$ & 90 \\
\hline$V\left[\AA^{3}\right]$ & $1053.1(4)$ & $2057.7(5)$ & $1625.3(8)$ & $3147.1(14)$ \\
\hline$Z$ & 2 & 4 & 2 & 4 \\
\hline$\rho_{\text {calcd }}\left[\mathrm{g} \mathrm{cm}^{-3}\right]$ & 1.522 & 1.558 & 2.135 & 1.875 \\
\hline$\theta$ range $\left[{ }^{\circ}\right]$ & $2-26$ & $2-26$ & $2-26$ & $2-25$ \\
\hline Measured reflections & 4490 & 4520 & 6941 & 6121 \\
\hline Unique reflections & 4129 & 4065 & 6429 & 5532 \\
\hline$R_{\text {int }}$ & 0.019 & 0.035 & 0.042 & 0.1197 \\
\hline Strong data $\left[\mathrm{I}_{\mathrm{O}}>2 \sigma\left(\mathrm{I}_{\mathrm{O}}\right)\right]$ & 3347 & 2394 & 5045 & 3540 \\
\hline Refined parameters & 307 & 307 & 397 & 401 \\
\hline$R 1, \mathrm{w} R 2$ (strong data) & $0.0403,0.1018$ & $0.0545,0.1029$ & $0.0761,0.1819$ & $0.1087,0.2012$ \\
\hline$R 1, \mathrm{w} R 2$ (all data) & $0.0530,0.1096$ & $0.1165,0.1243$ & $0.0999,0.2077$ & $0.1693,0.2417$ \\
\hline GoF & 1.024 & 1.007 & 1.151 & 1.192 \\
\hline Max/min residuals $\left[\mathrm{e} \AA^{-3}\right]$ & $0.42 /-0.30$ & $0.26 /-0.25$ & $1.08 /-1.22$ & $0.70 /-0.67$ \\
\hline
\end{tabular}




\section{Copies of ${ }^{1} \mathrm{H},{ }^{13} \mathrm{C}$ NMR, ESI-MS and GC-MS spectra}

Compound 2

${ }^{1} \mathrm{H} \mathrm{NMR}\left(200 \mathrm{MHz}, \mathrm{CDCl}_{3}\right)$

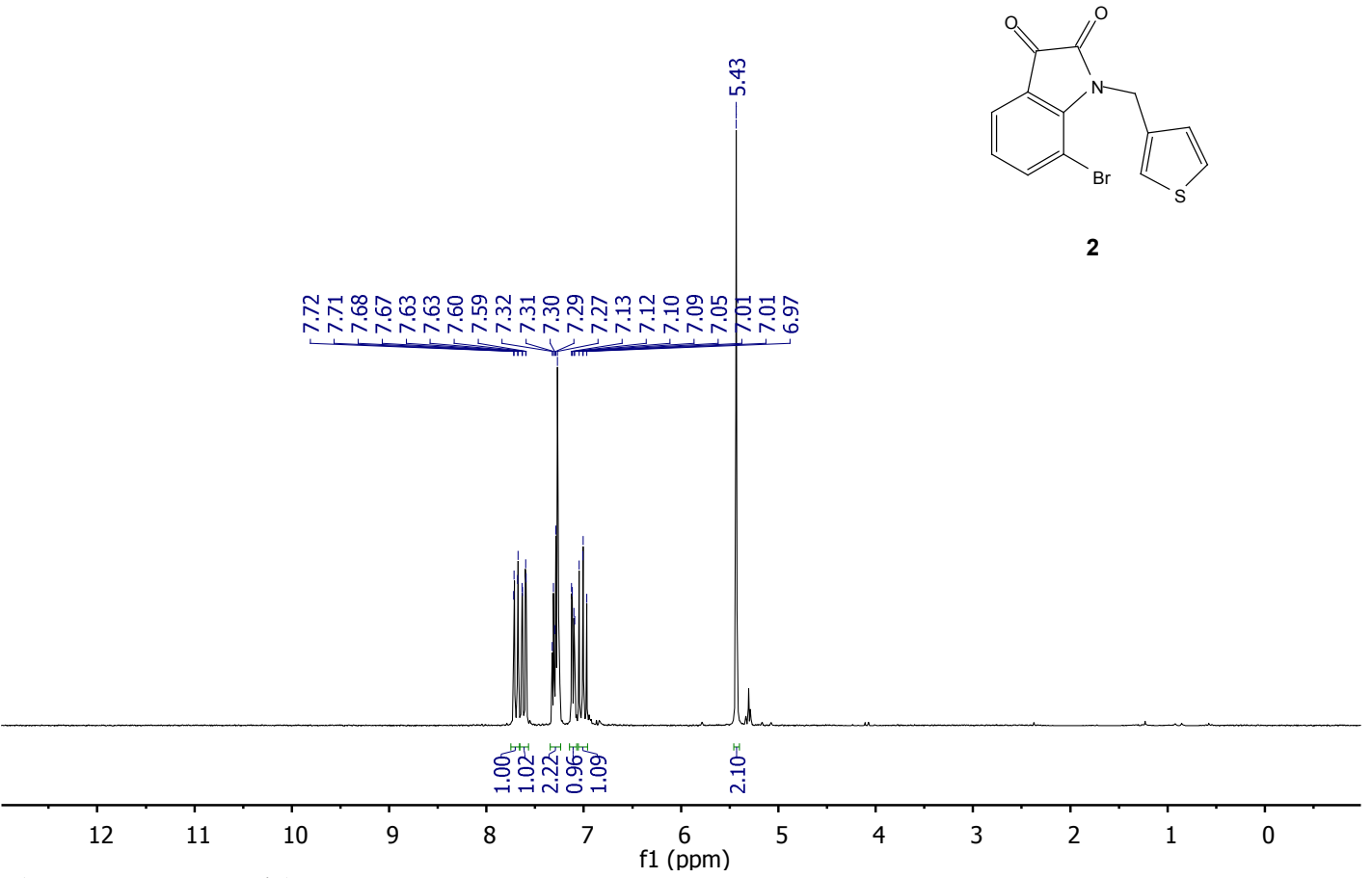

${ }^{13} \mathrm{C}$ NMR $\left(75 \mathrm{MHz}, \mathrm{CDCl}_{3}\right)$
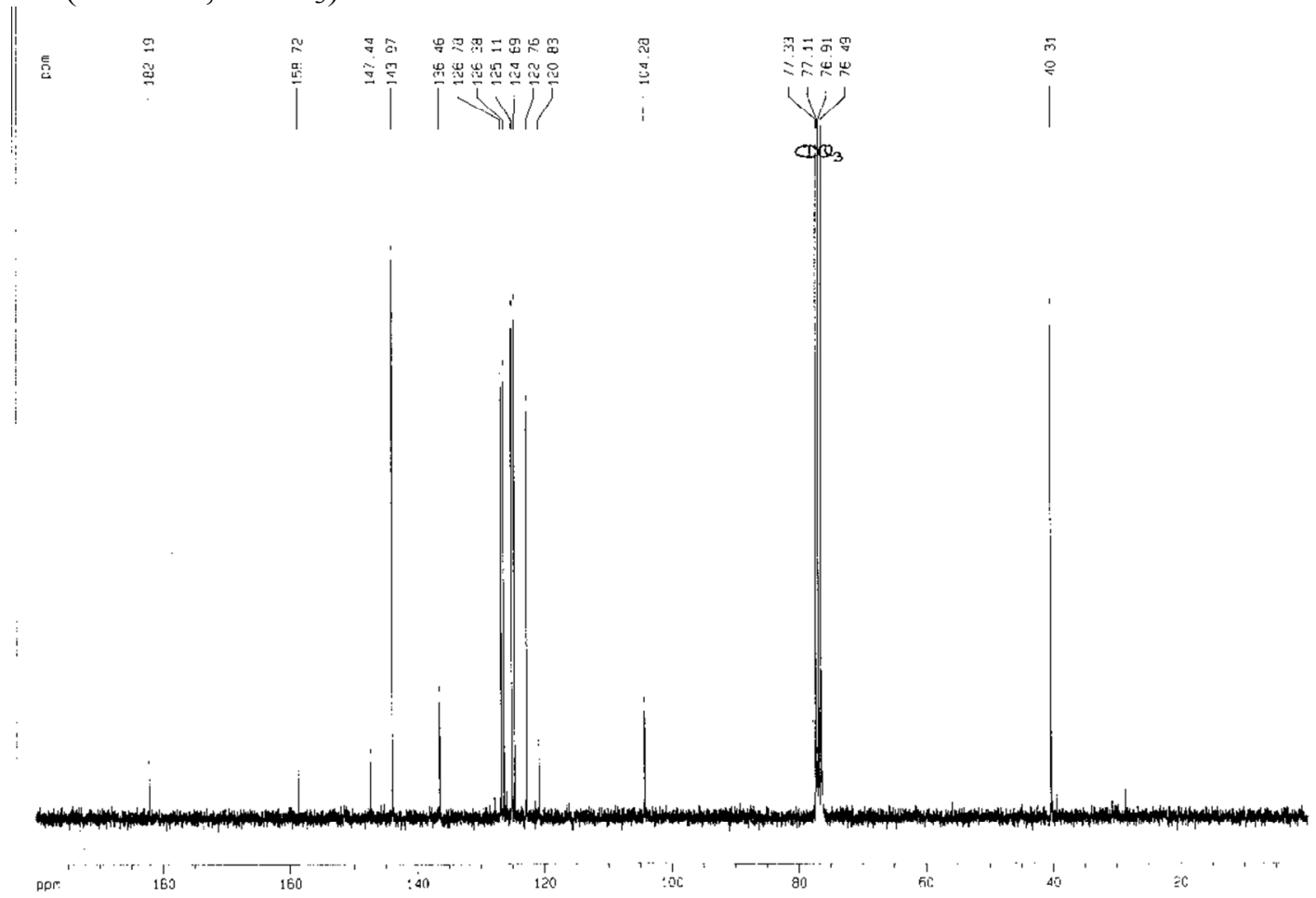


\section{GC-MS}

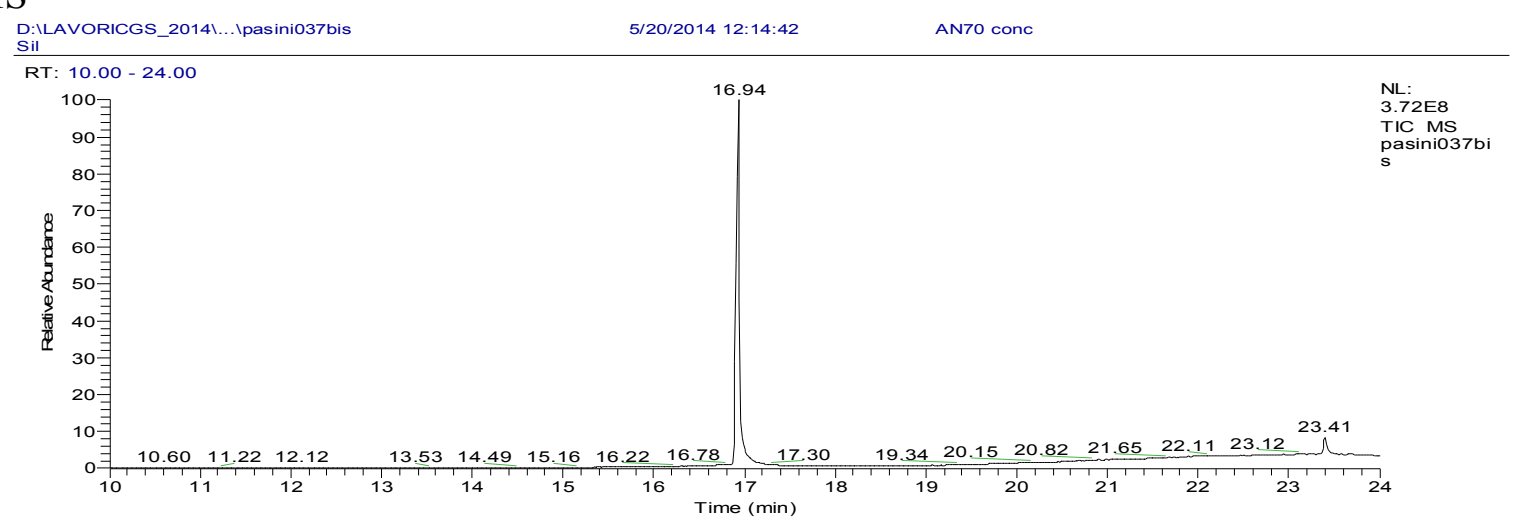

pasiniO37bis \#1125 RT: 16.90 AV: 1 NL: 2.12E7

96.99

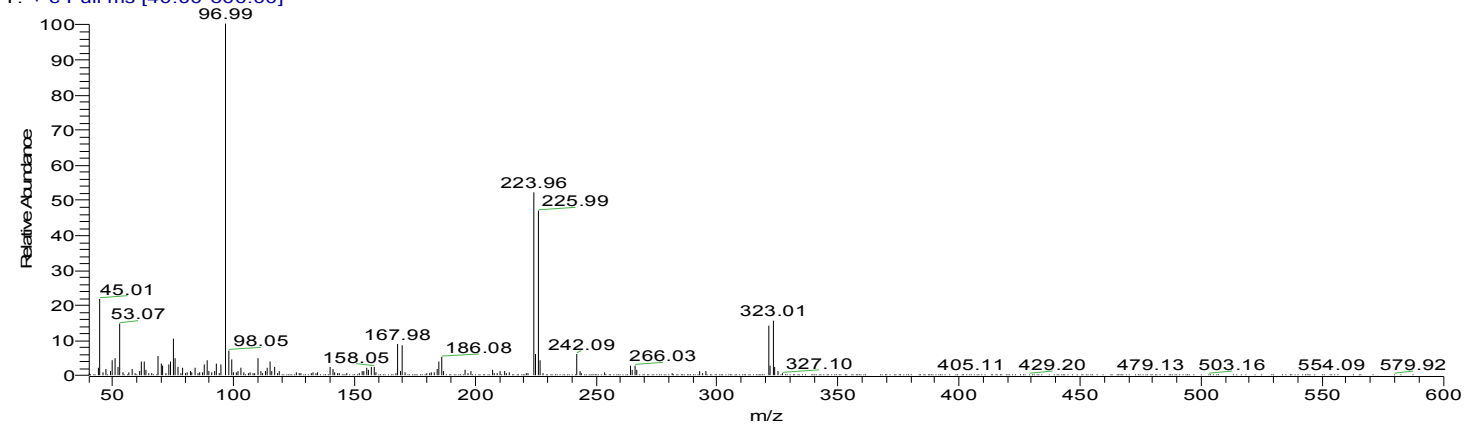


Compound 3

${ }^{1} \mathrm{H}$ NMR $\left(200 \mathrm{MHz}, \mathrm{CDCl}_{3}\right)$

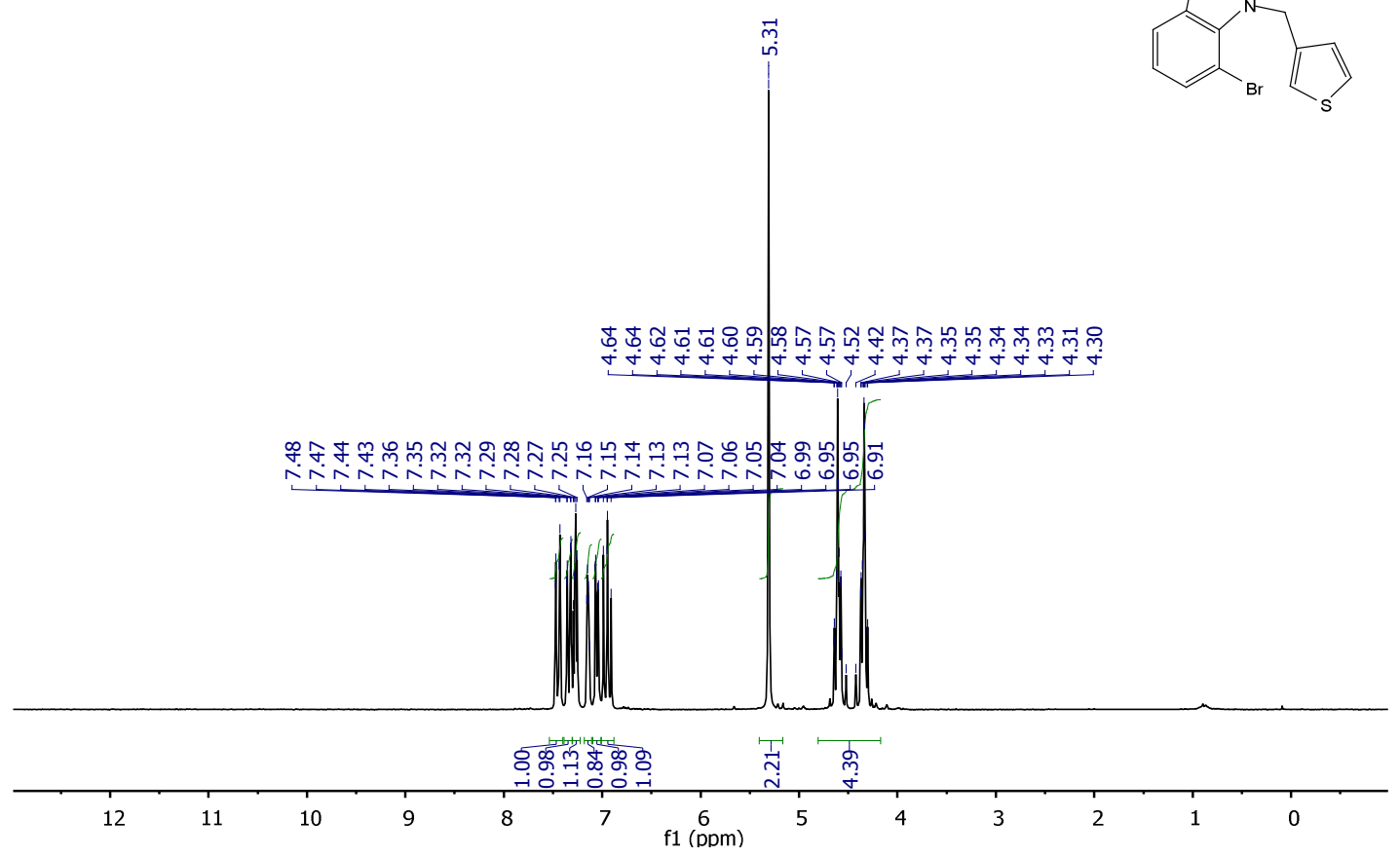

${ }^{13} \mathrm{C}$ NMR $\left(75 \mathrm{MHz}, \mathrm{CDCl}_{3}\right)$

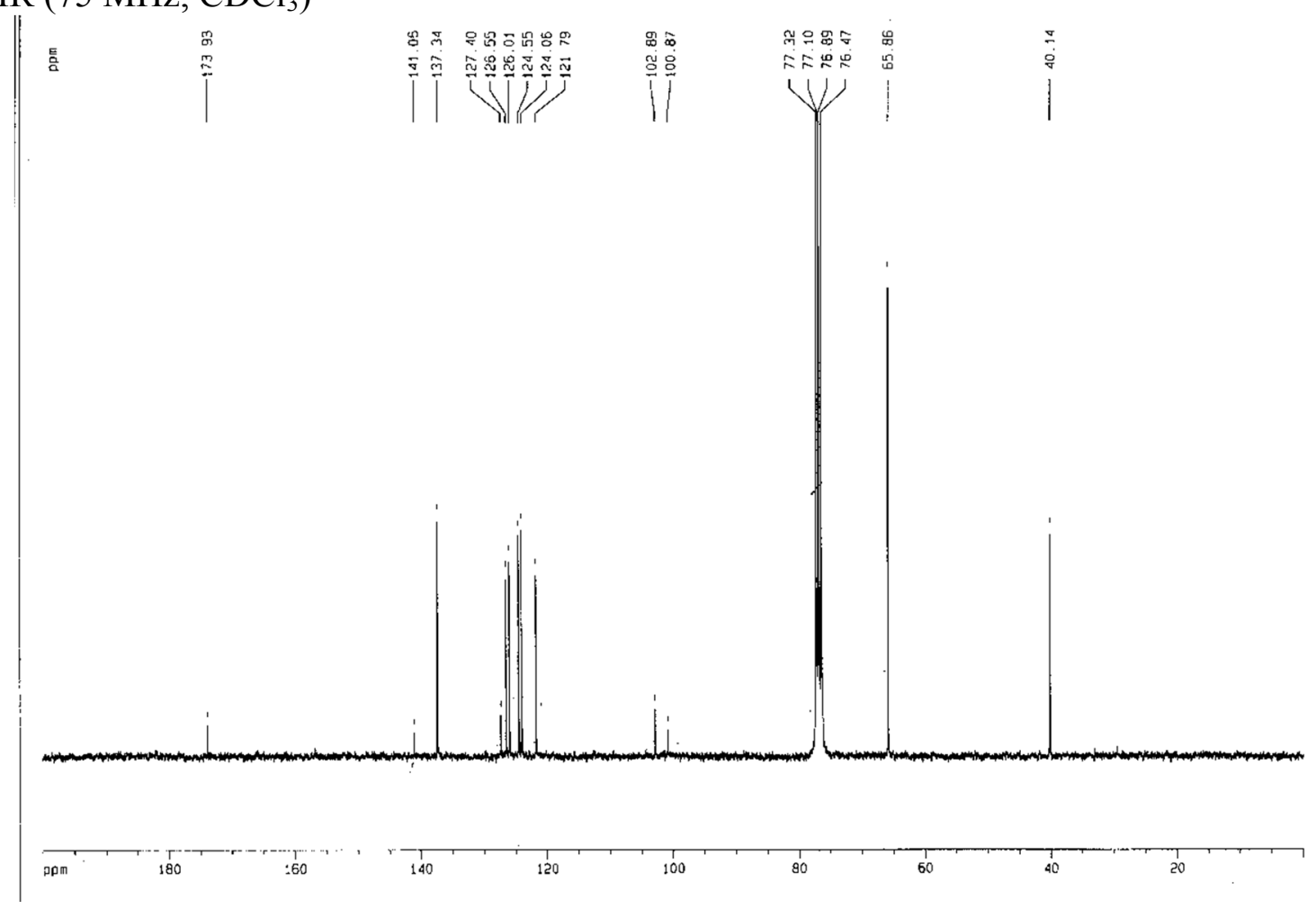




\section{GCMS}

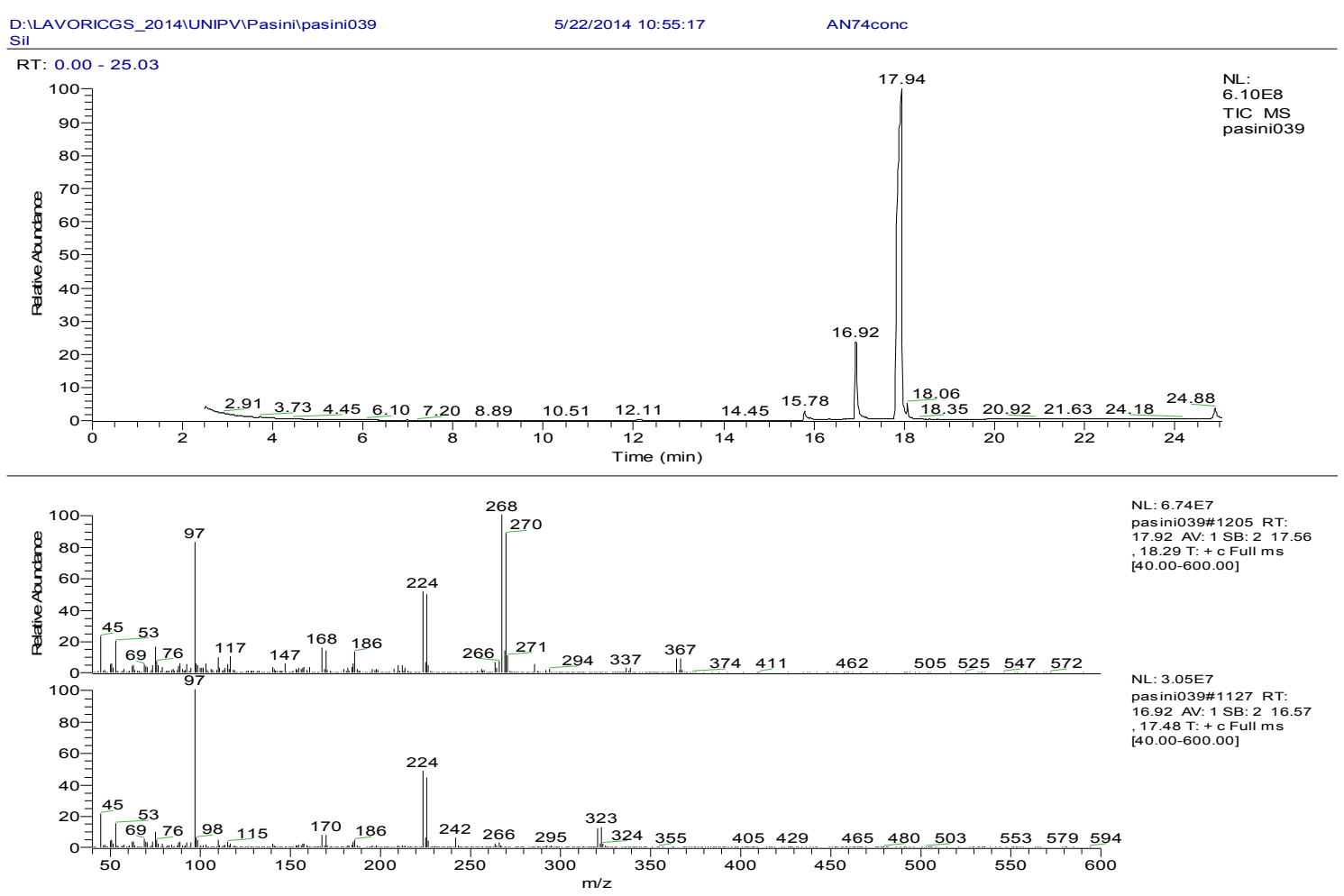




\section{Compound 4}

${ }^{1} \mathrm{H}$ NMR $\left(200 \mathrm{MHz}, \mathrm{CDCl}_{3}\right)$

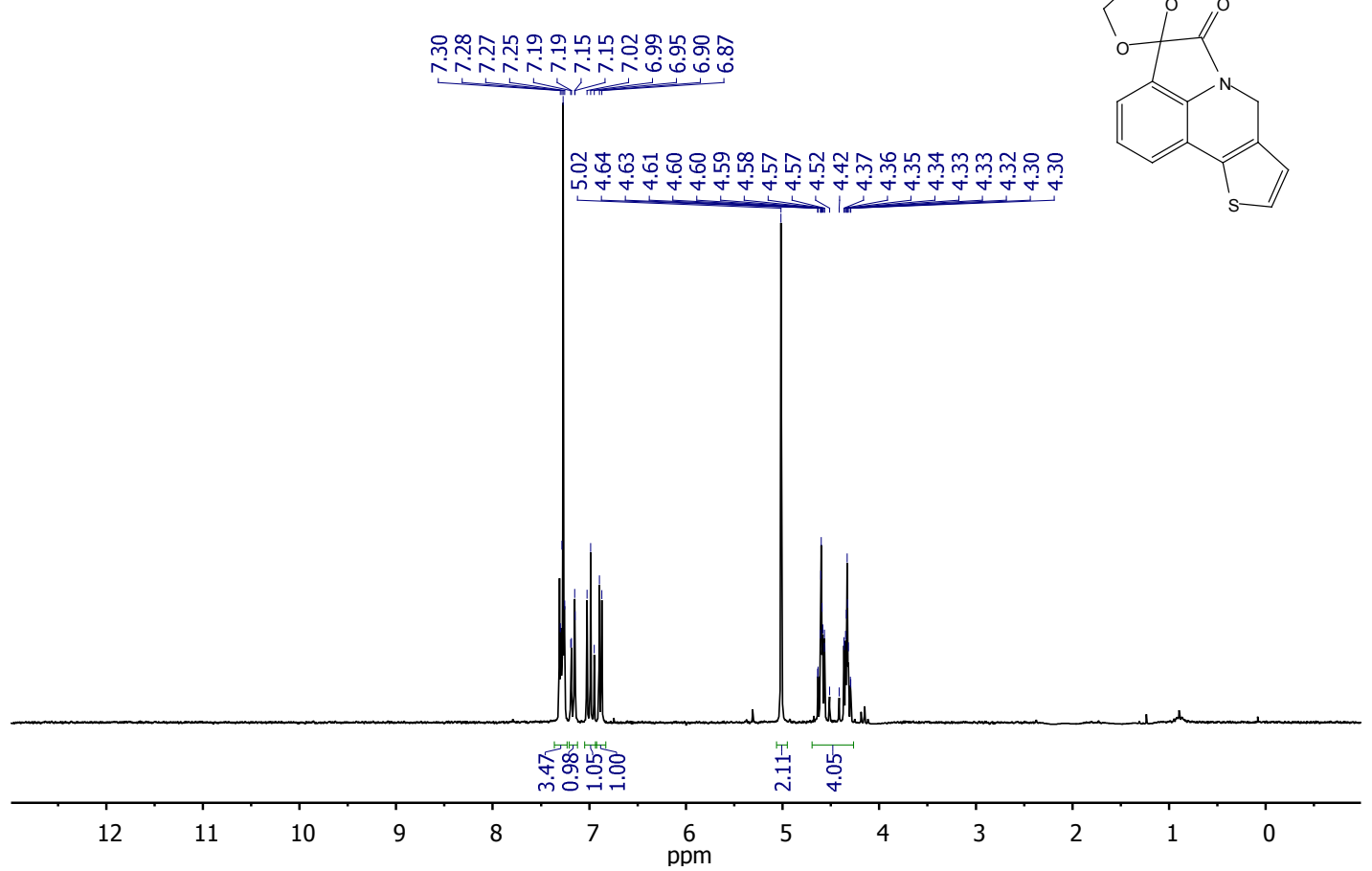

${ }^{13} \mathrm{C}$ NMR $\left(75 \mathrm{MHz}, \mathrm{CDCl}_{3}\right.$ )

言

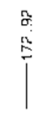

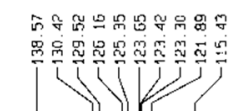

i
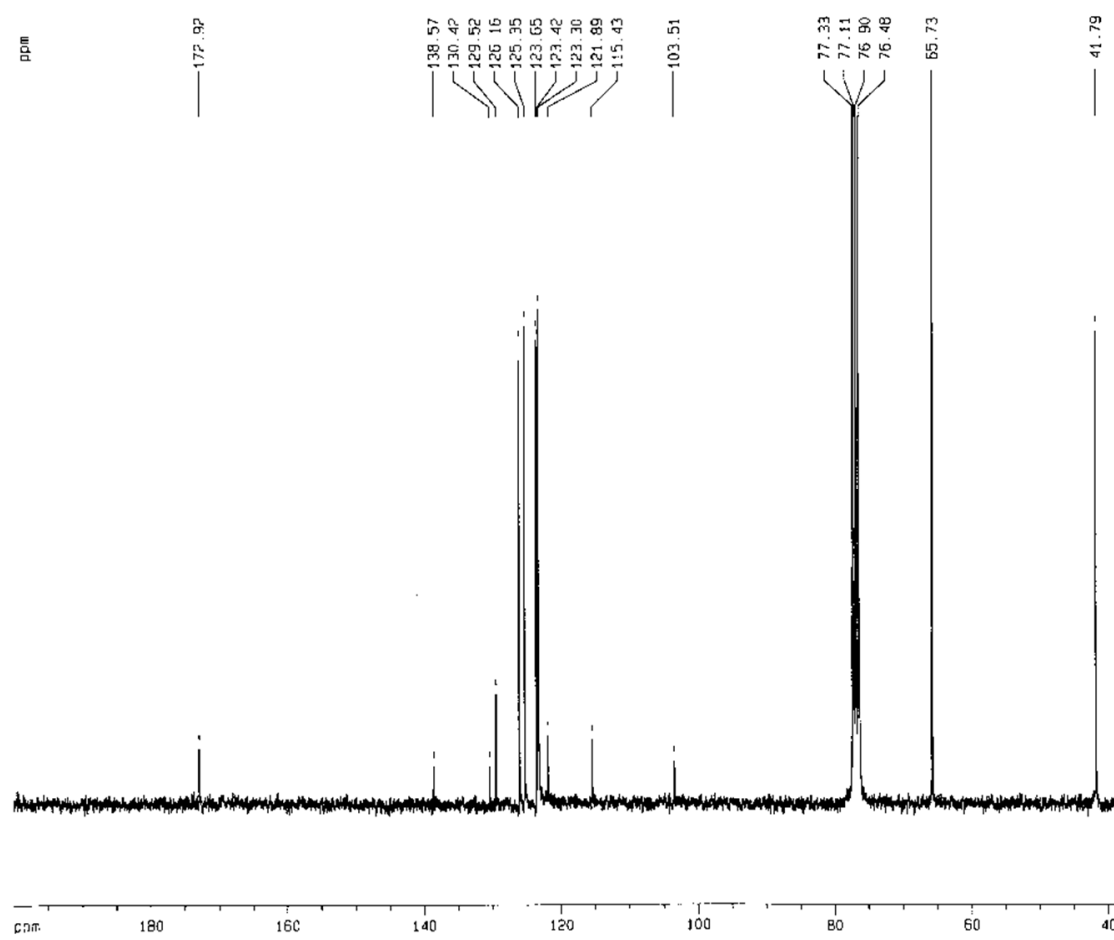

100
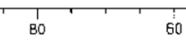


\section{GCMS}

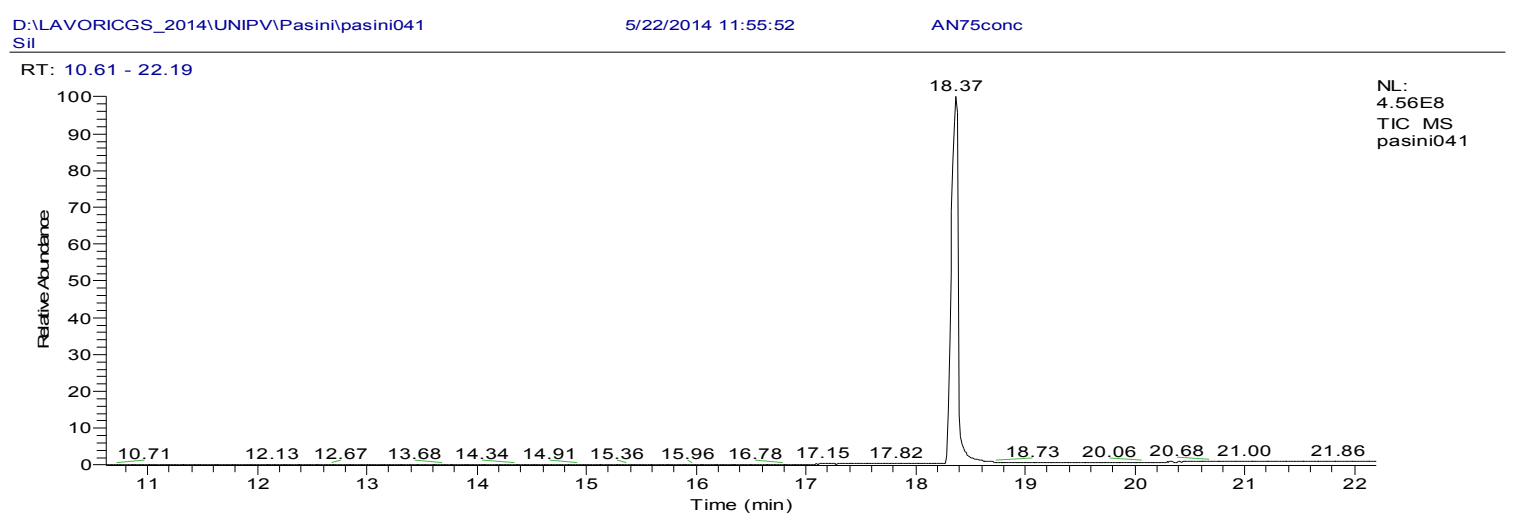

pasiniO41 \#1238 RT: 18.35 AV: 1 SB: 2 18.19, 18.97 NL: 6.76E7

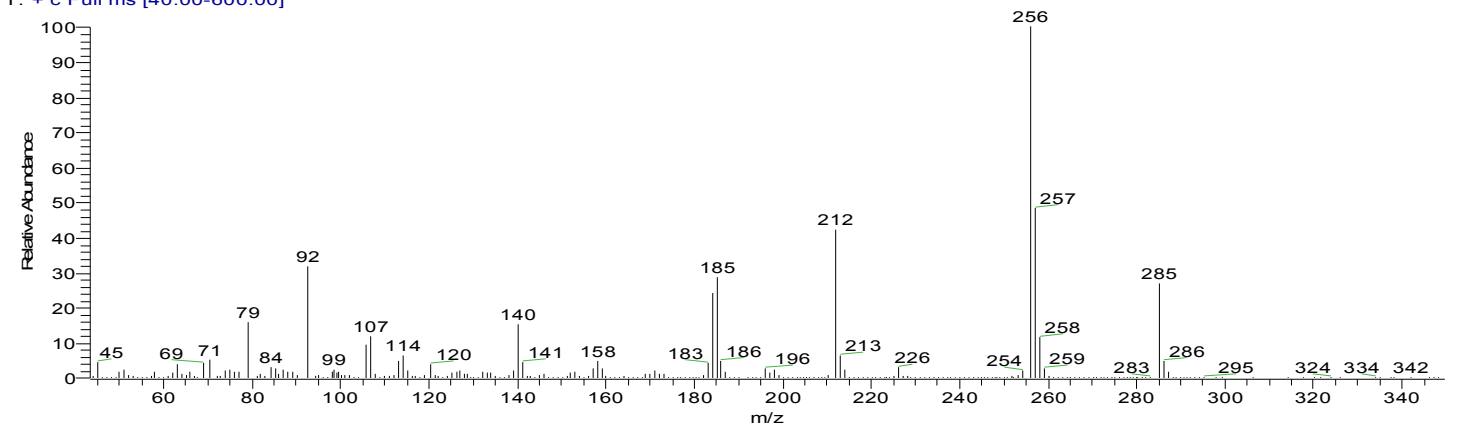


Compound 5

${ }^{1} \mathrm{H}$ NMR (200 MHz, $\mathrm{CDCl}_{3}$ )

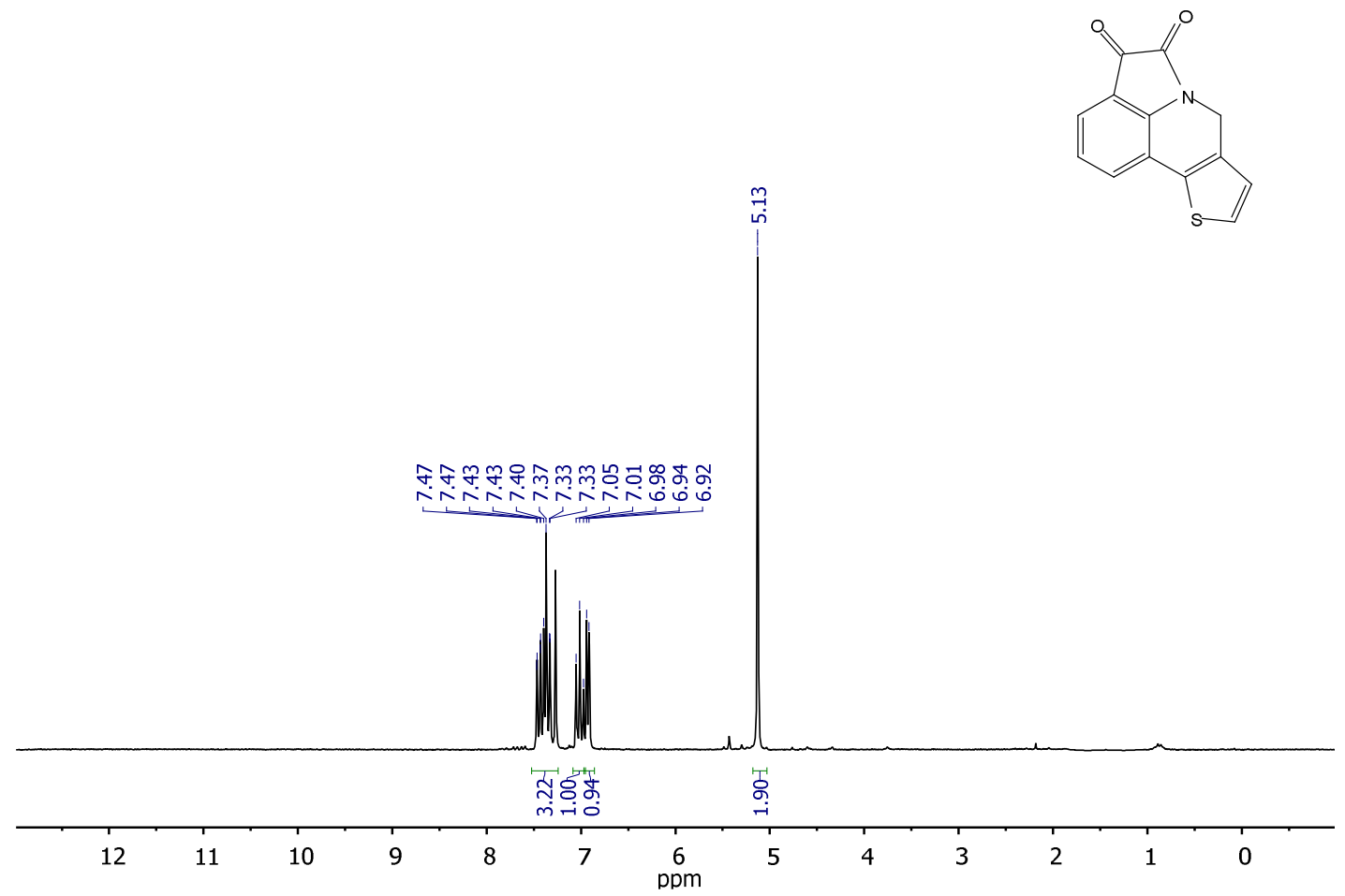

${ }^{13} \mathrm{C}$ NMR $\left(75 \mathrm{MHz}, \mathrm{CDCl}_{3}\right)$

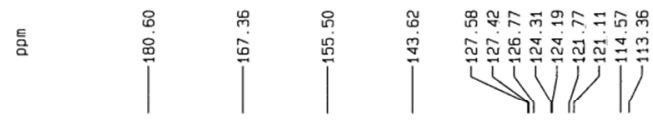
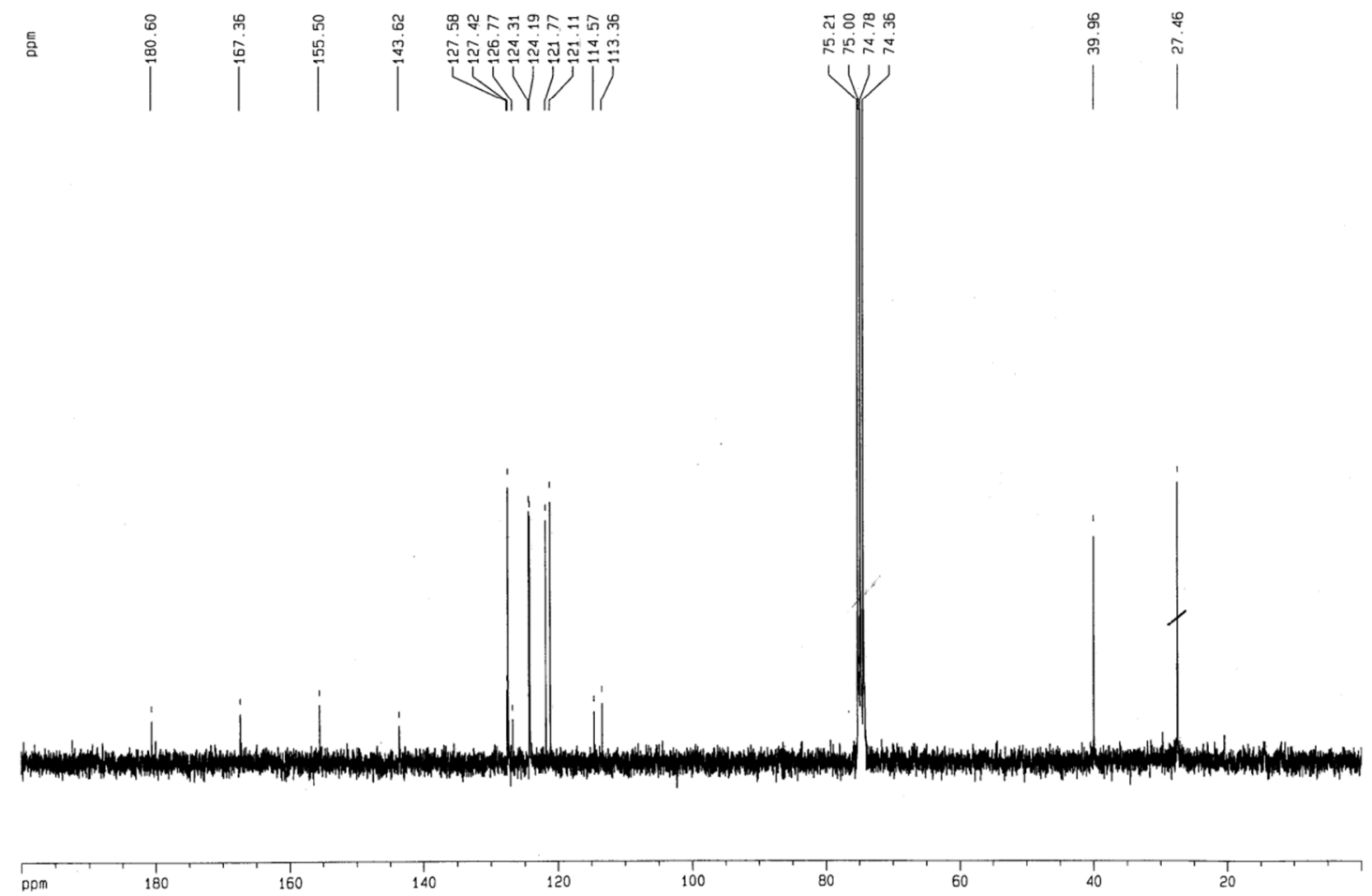


\section{ESI-MS}

MS12a \#300-354 RT: 4.17-4.86 AV: 55 NL: 1.36E3

T: ITMS + c ESI Full ms [150.00-2000.00]

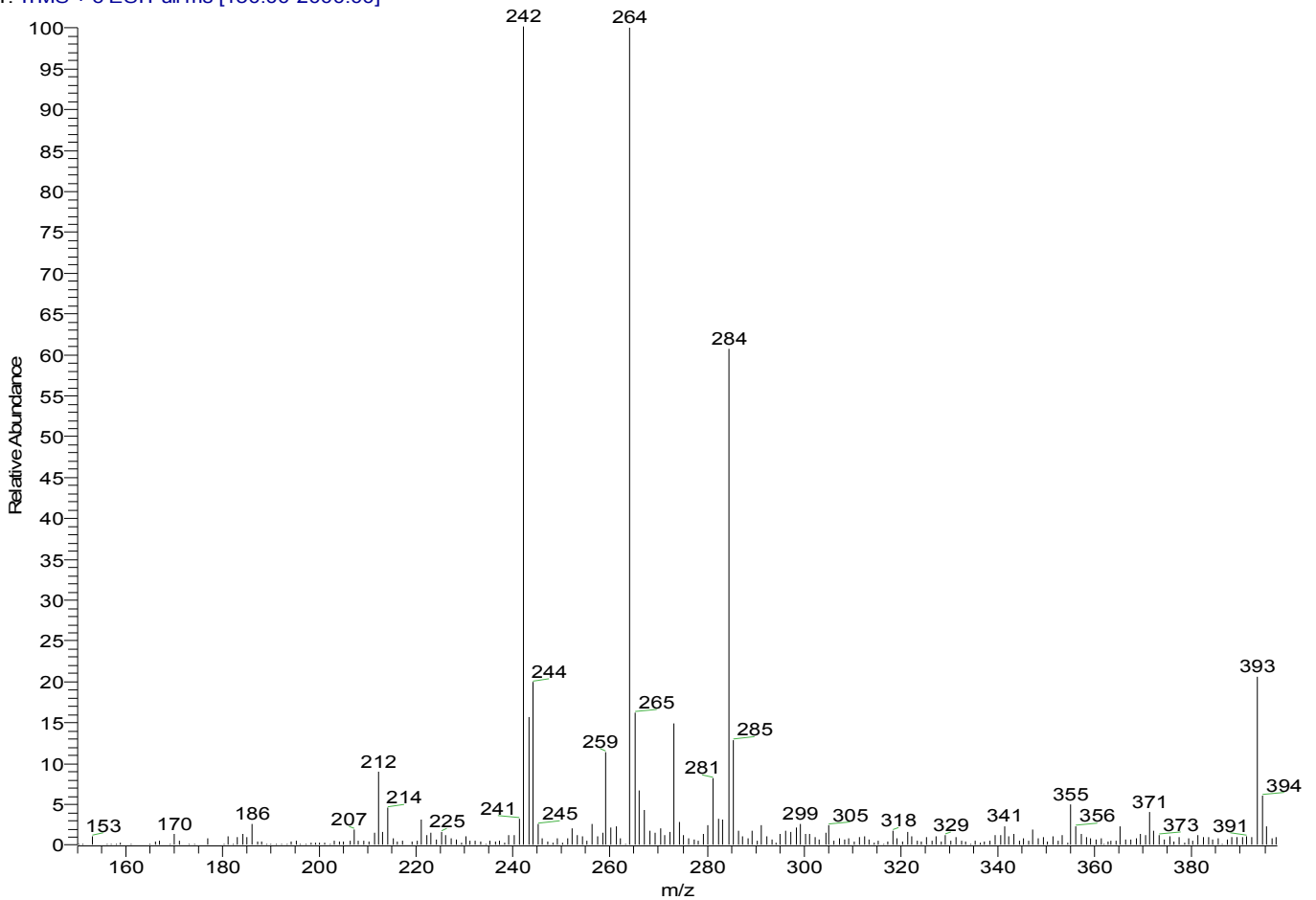


Compound 7

${ }^{1} \mathrm{H}$ NMR $\left(200 \mathrm{MHz}, \mathrm{CDCl}_{3}\right)$
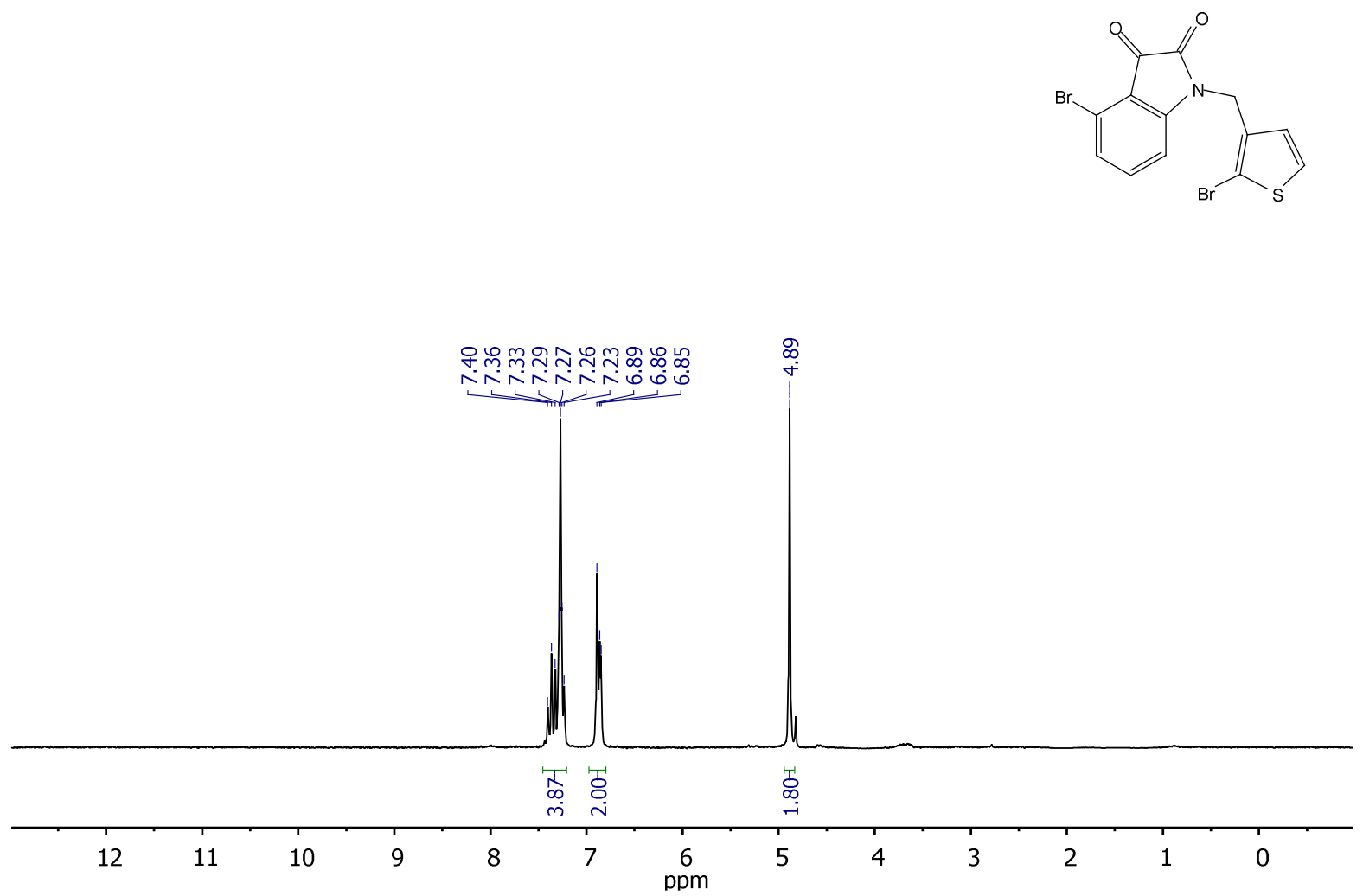

${ }^{13} \mathrm{C}$ NMR $\left(75 \mathrm{MHz}, \mathrm{CDCl}_{3}\right.$ )
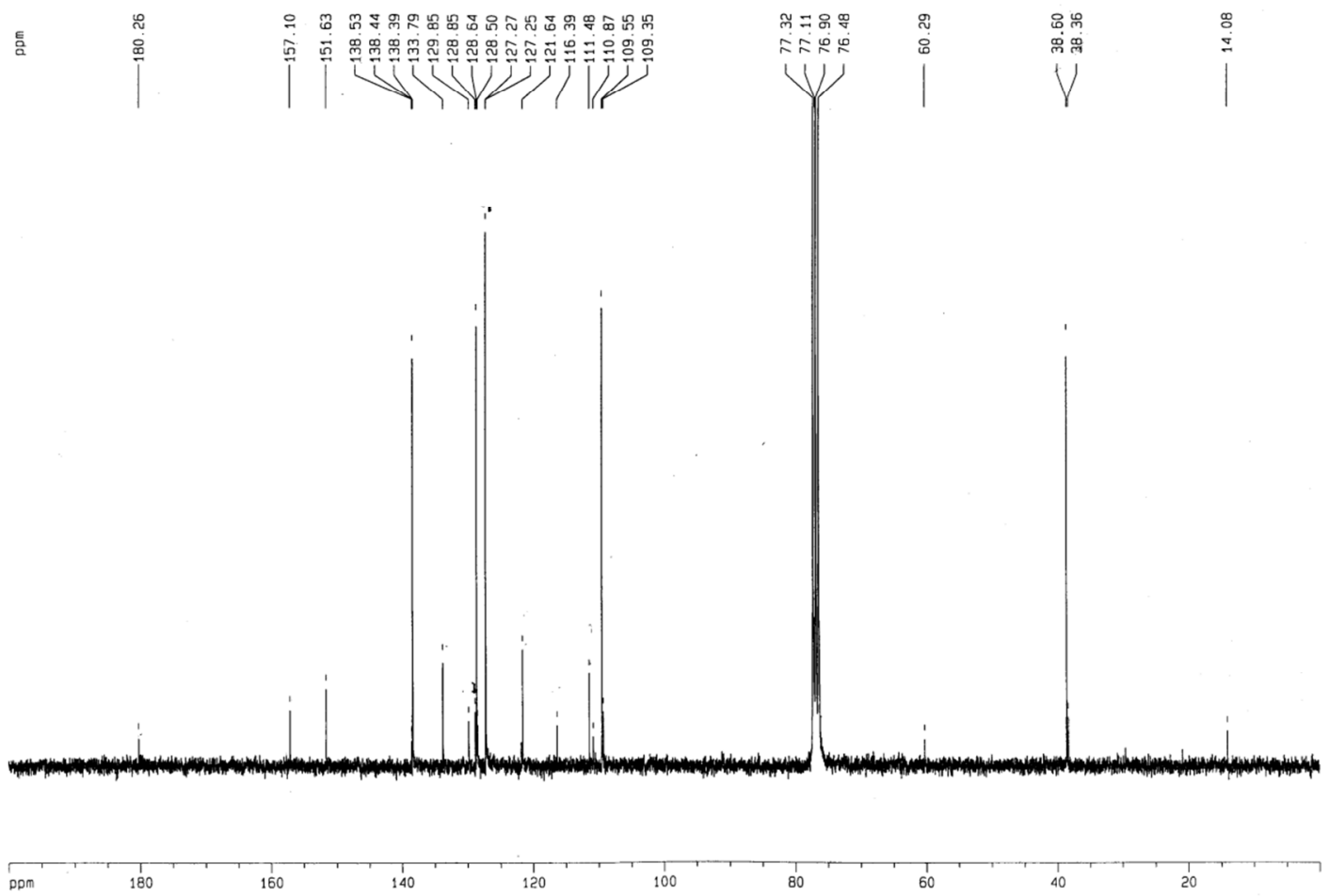


\section{Compound 8}

${ }^{1} \mathrm{H}$ NMR (200 MHz, $\mathrm{CDCl}_{3}$ )
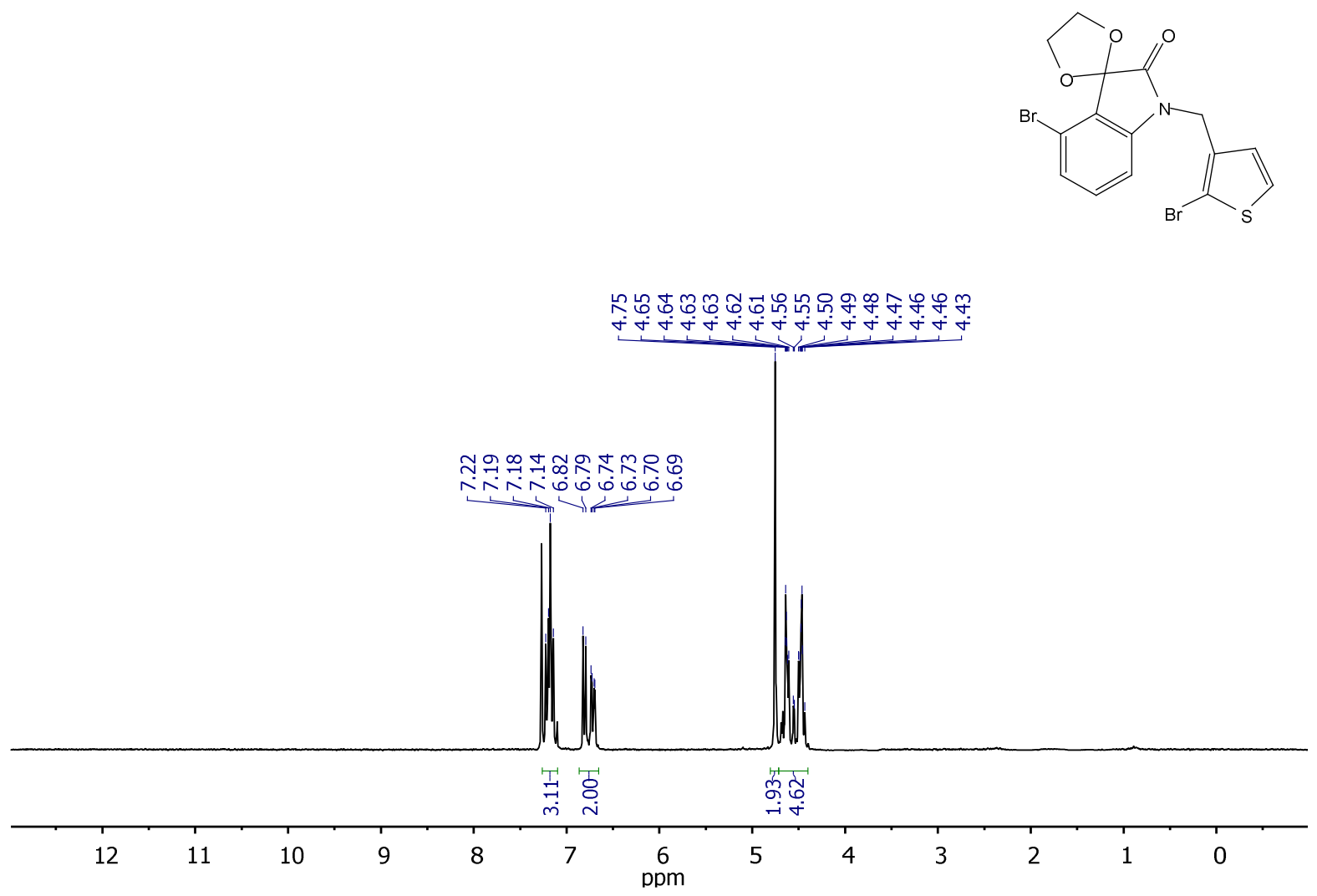

${ }^{13} \mathrm{C}$ NMR (75 MHz, $\mathrm{CDCl}_{3}$ )

⿷

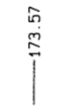

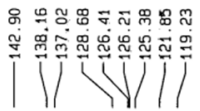

V

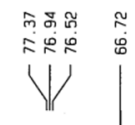

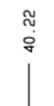

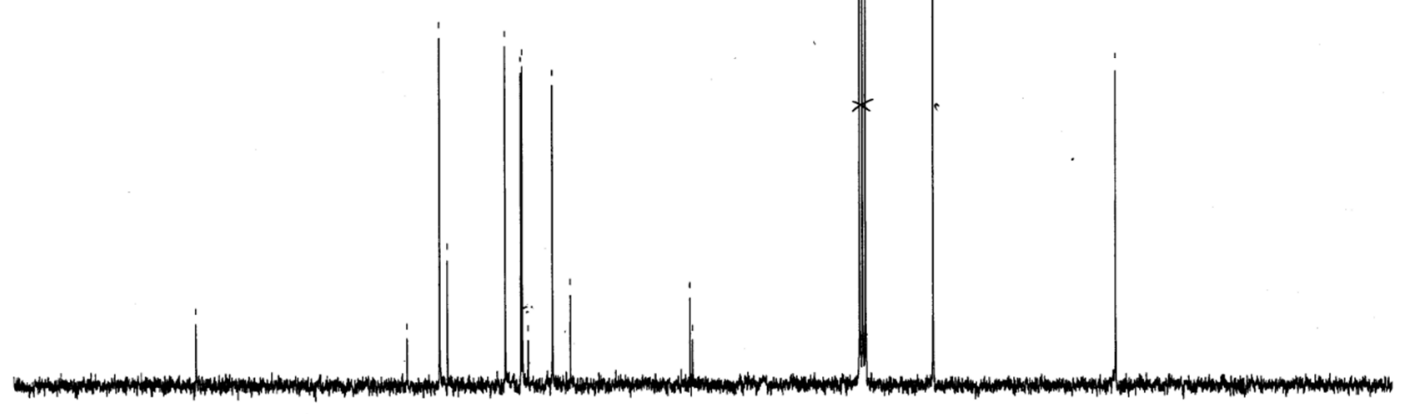

ppm

180

160

140

120

80

20 
Compound 12

${ }^{1} \mathrm{H}$ NMR (200 MHz, $\mathrm{CDCl}_{3}$ )

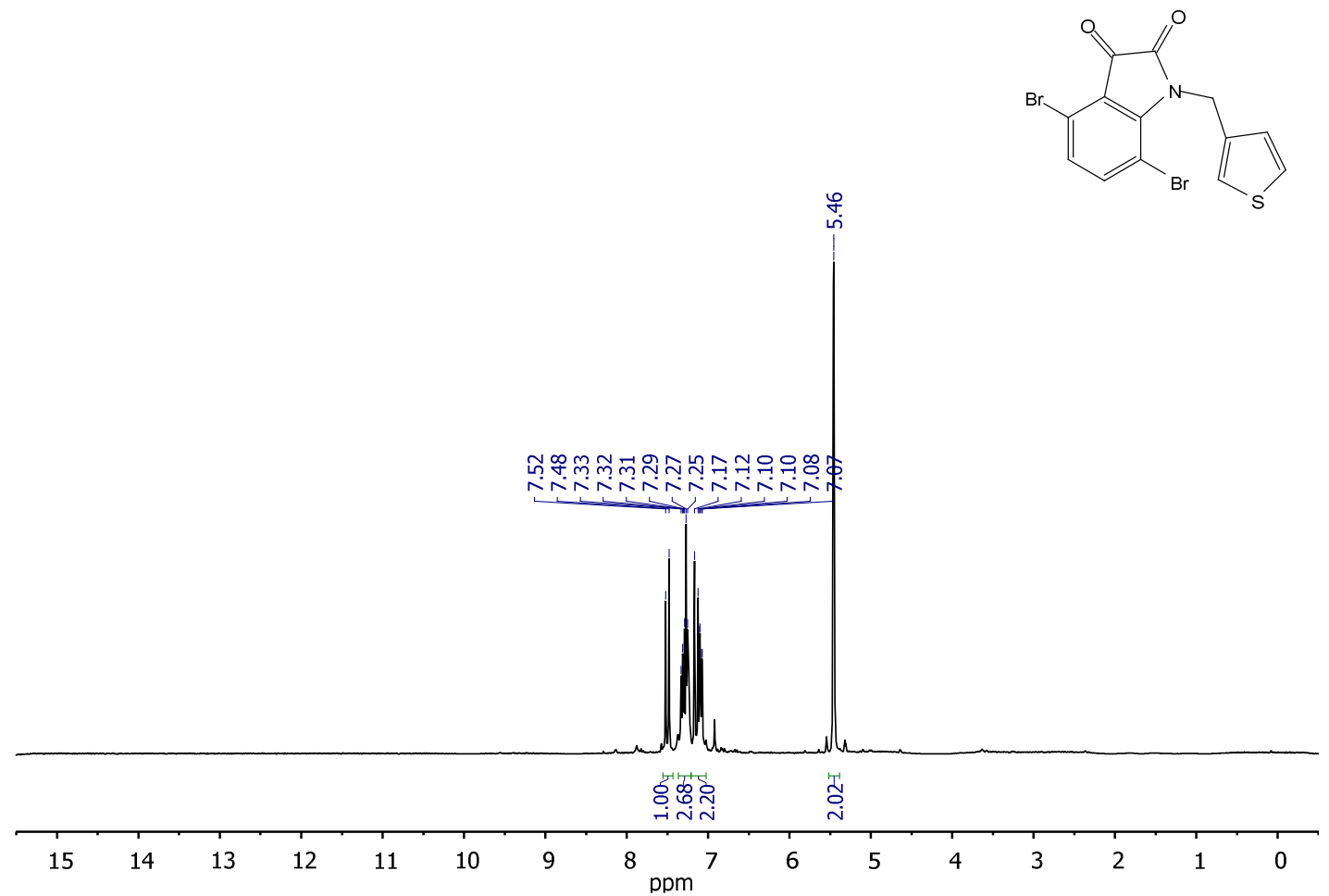

${ }^{13} \mathrm{C}$ NMR (75 MHz, $\mathrm{CDCl}_{3}$ )
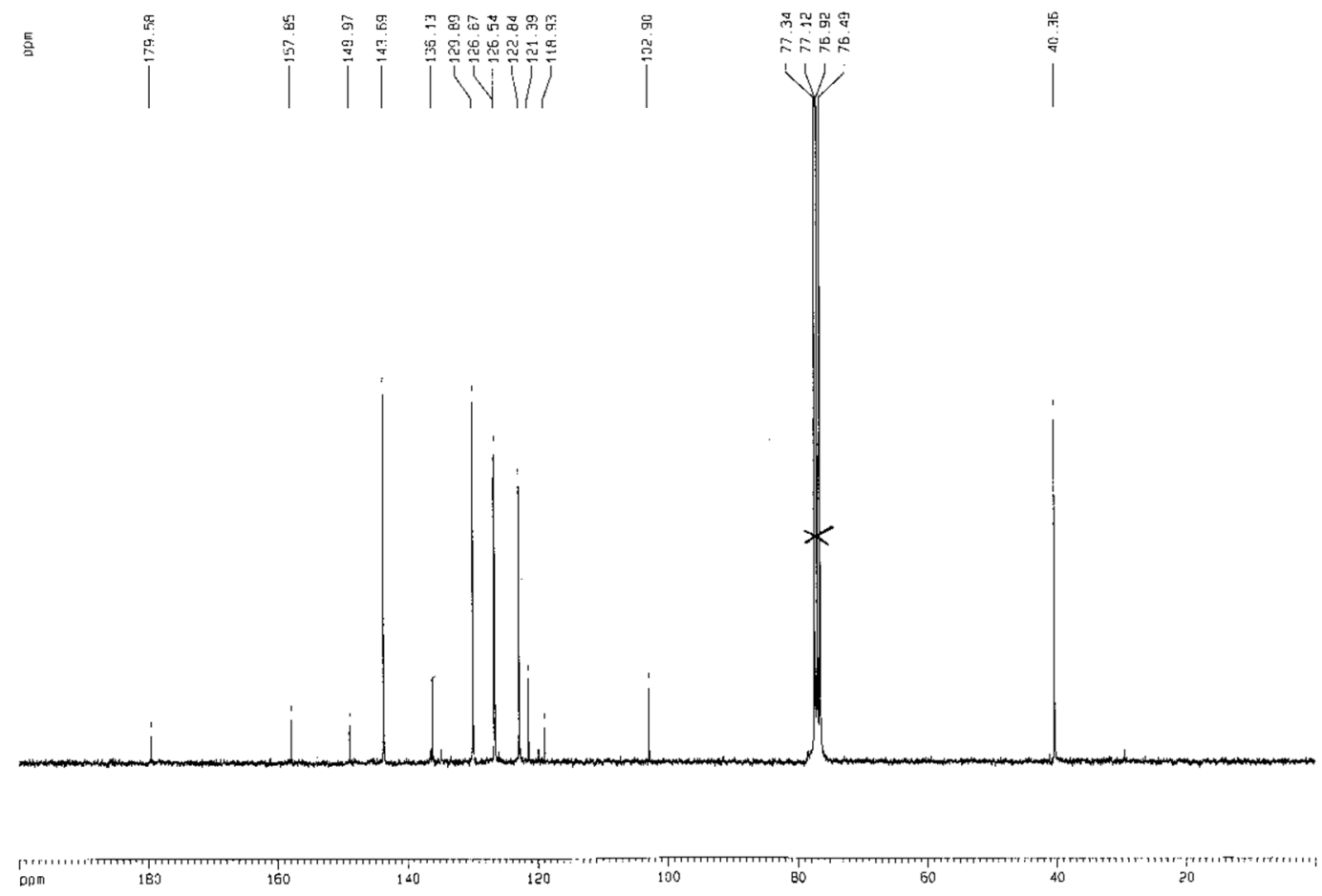


\section{Compound 13}

${ }^{1} \mathrm{H}$ NMR (200 MHz, $\mathrm{CDCl}_{3}$ )
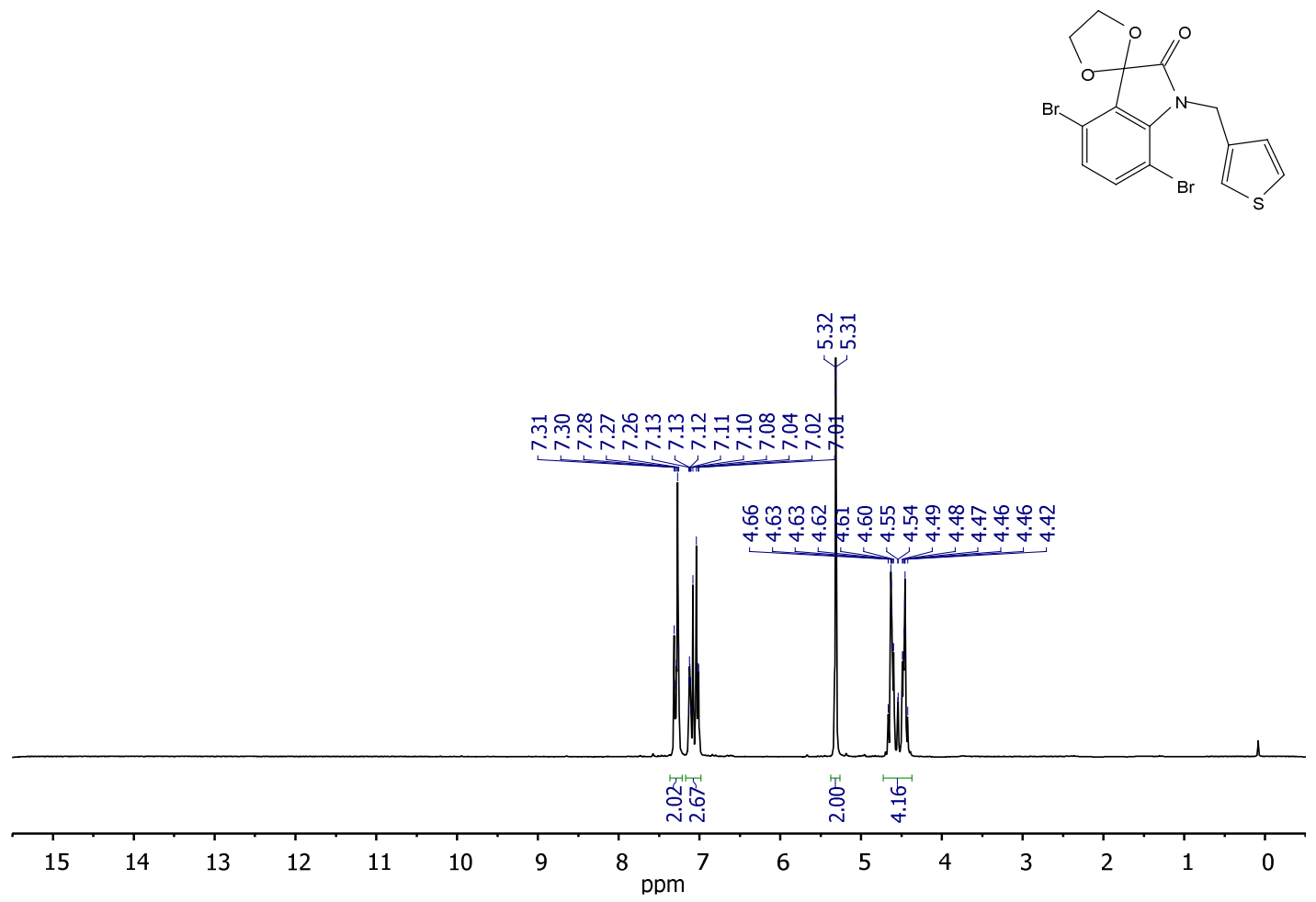

${ }^{13} \mathrm{C}$ NMR (75 MHz, $\mathrm{CDCl}_{3}$ )

⿷

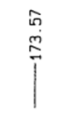

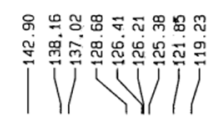

V

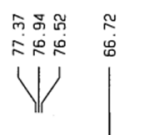

$\mid$
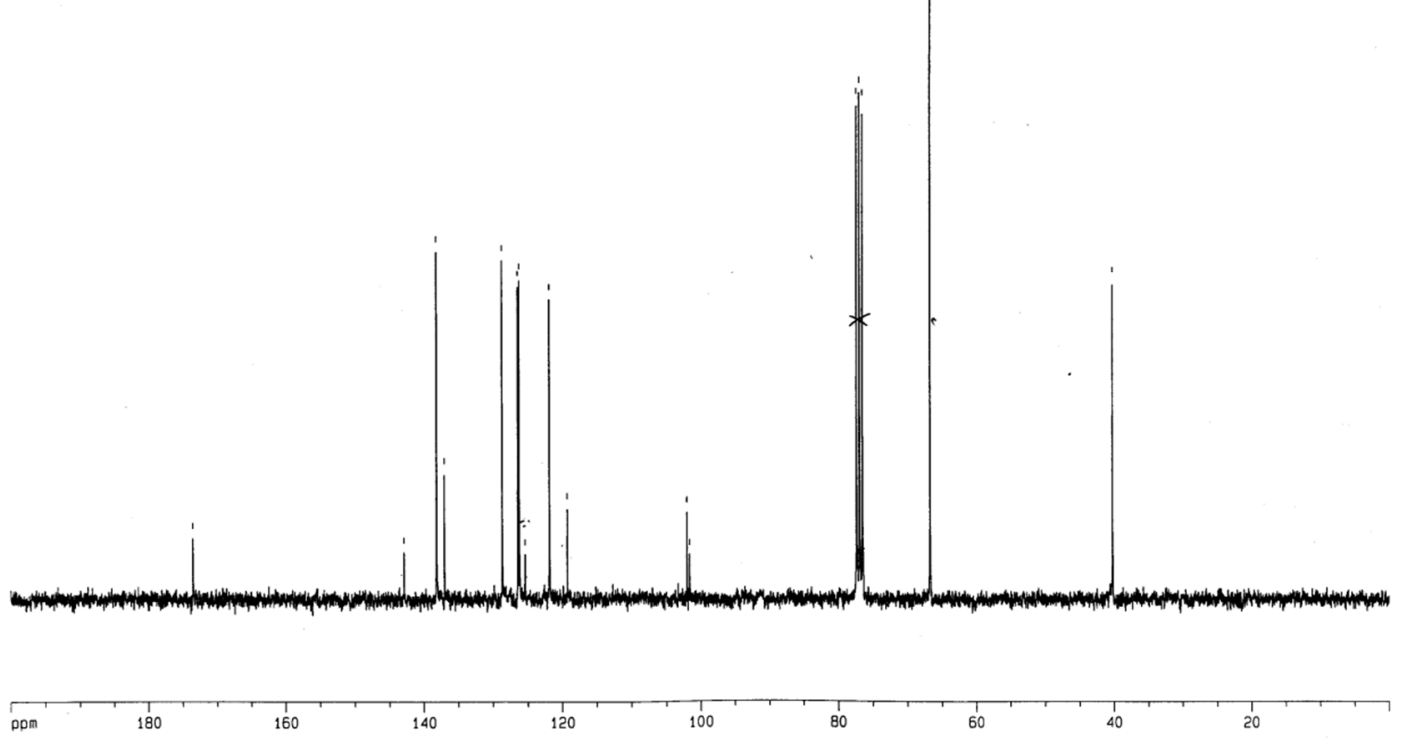


\section{Compound 15}

${ }^{1} \mathrm{H}$ NMR (200 MHz, $\mathrm{CDCl}_{3}$ )
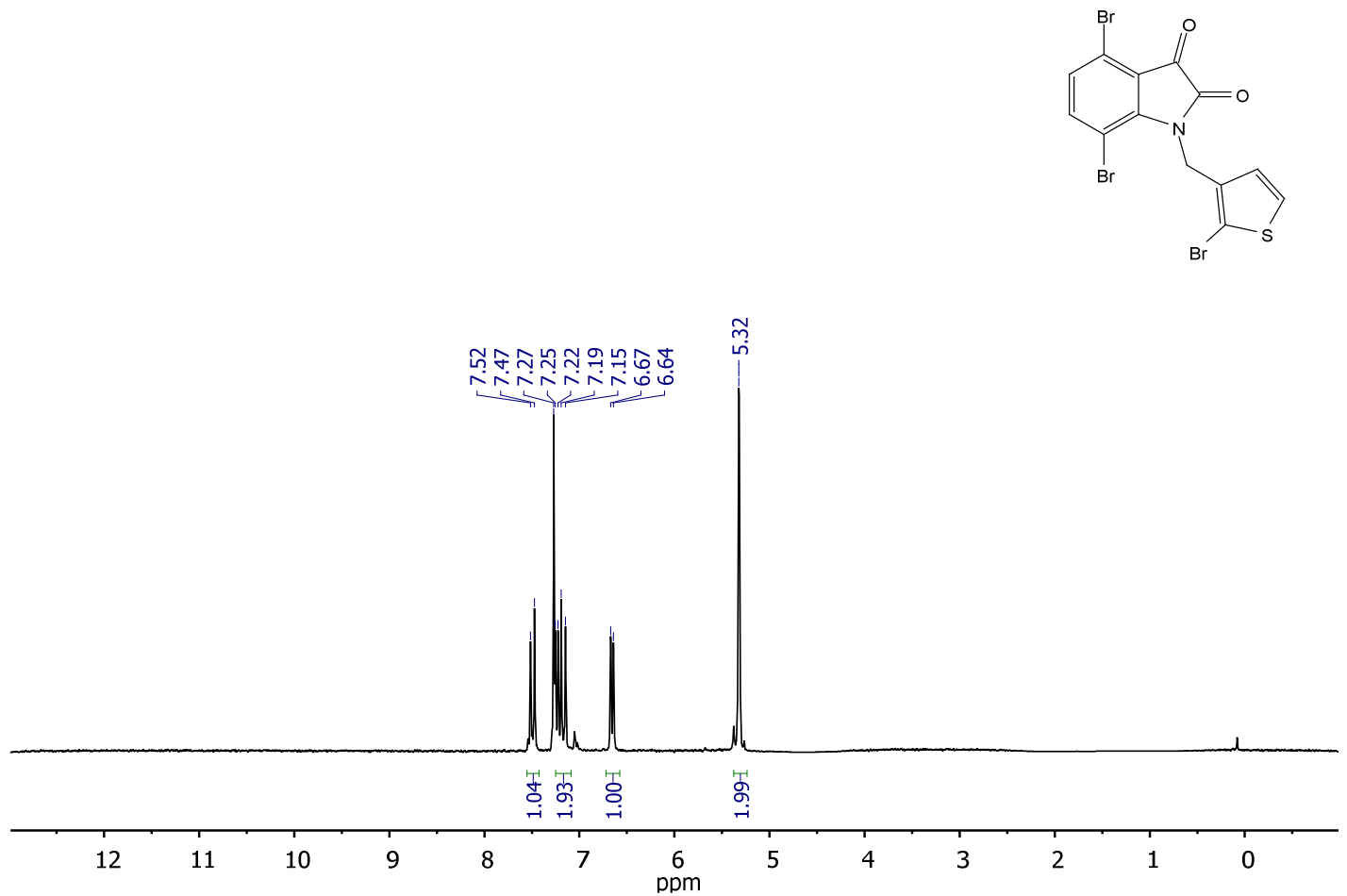

${ }^{13} \mathrm{C} \mathrm{NMR}\left(75 \mathrm{MHz}, \mathrm{CDCl}_{3}\right)$
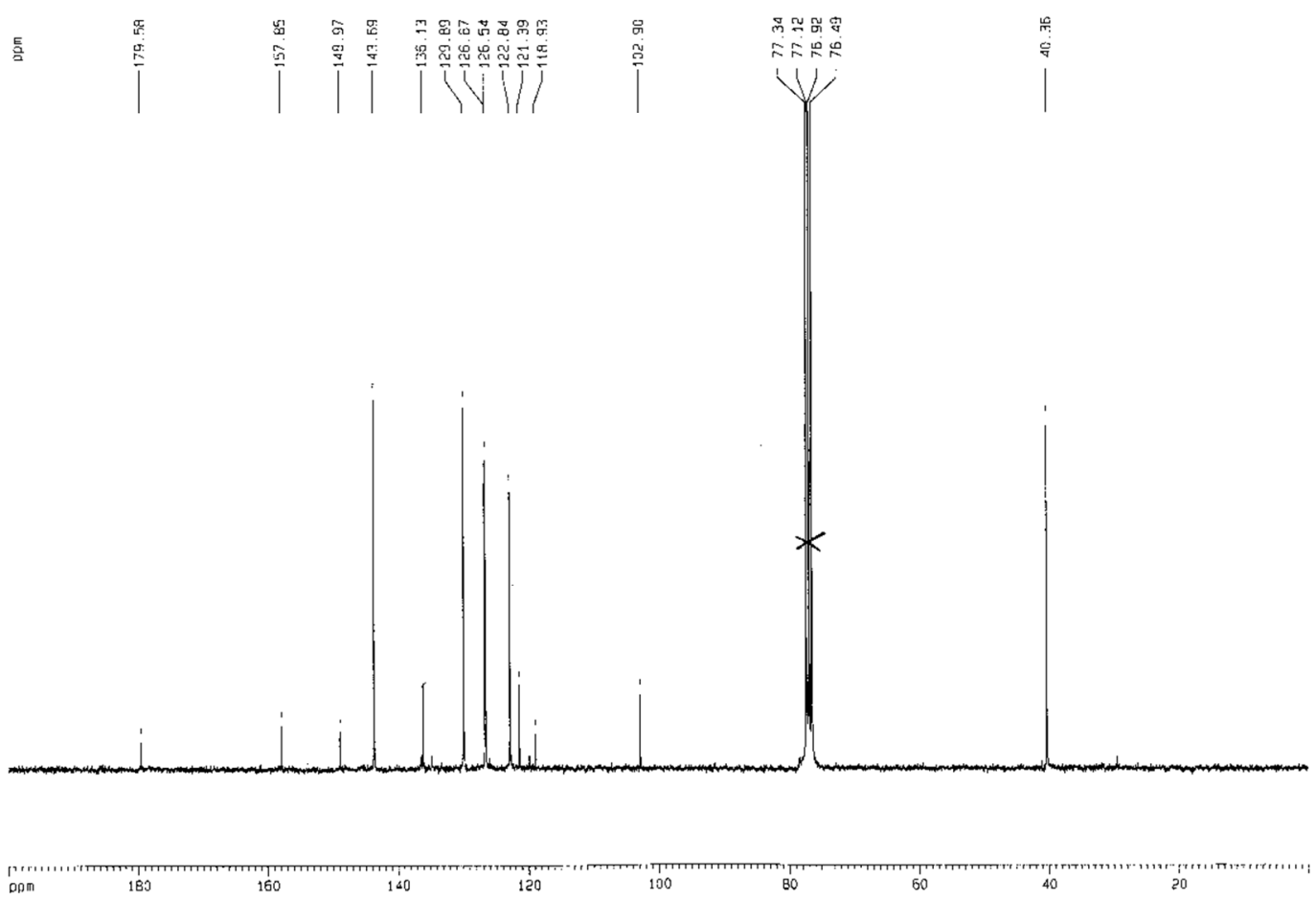
Compound 16

${ }^{1} \mathrm{H}$ NMR (200 MHz, $\mathrm{CDCl}_{3}$ )

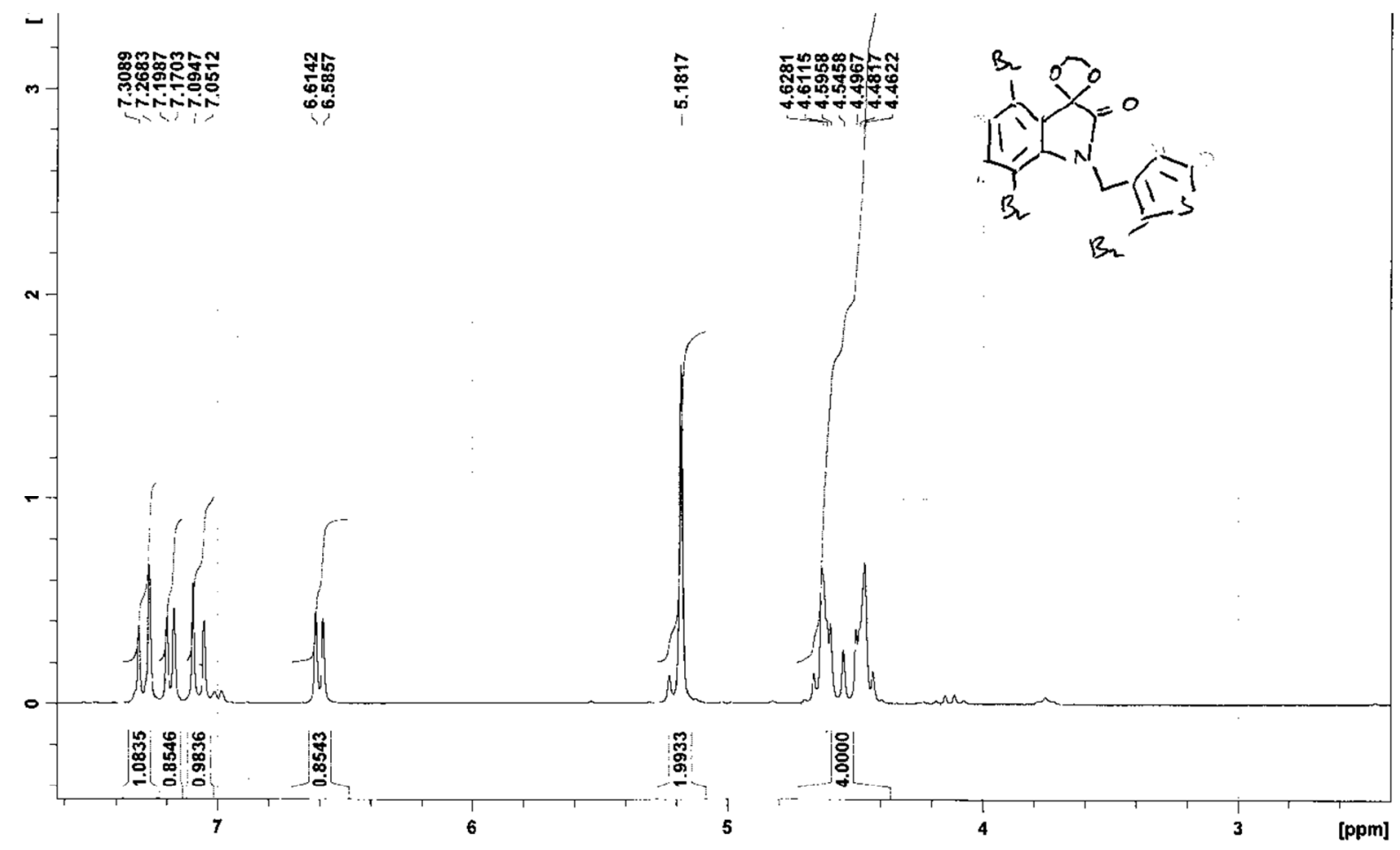

${ }^{13} \mathrm{C}$ NMR (75 MHz, $\mathrm{CDCl}_{3}$ )
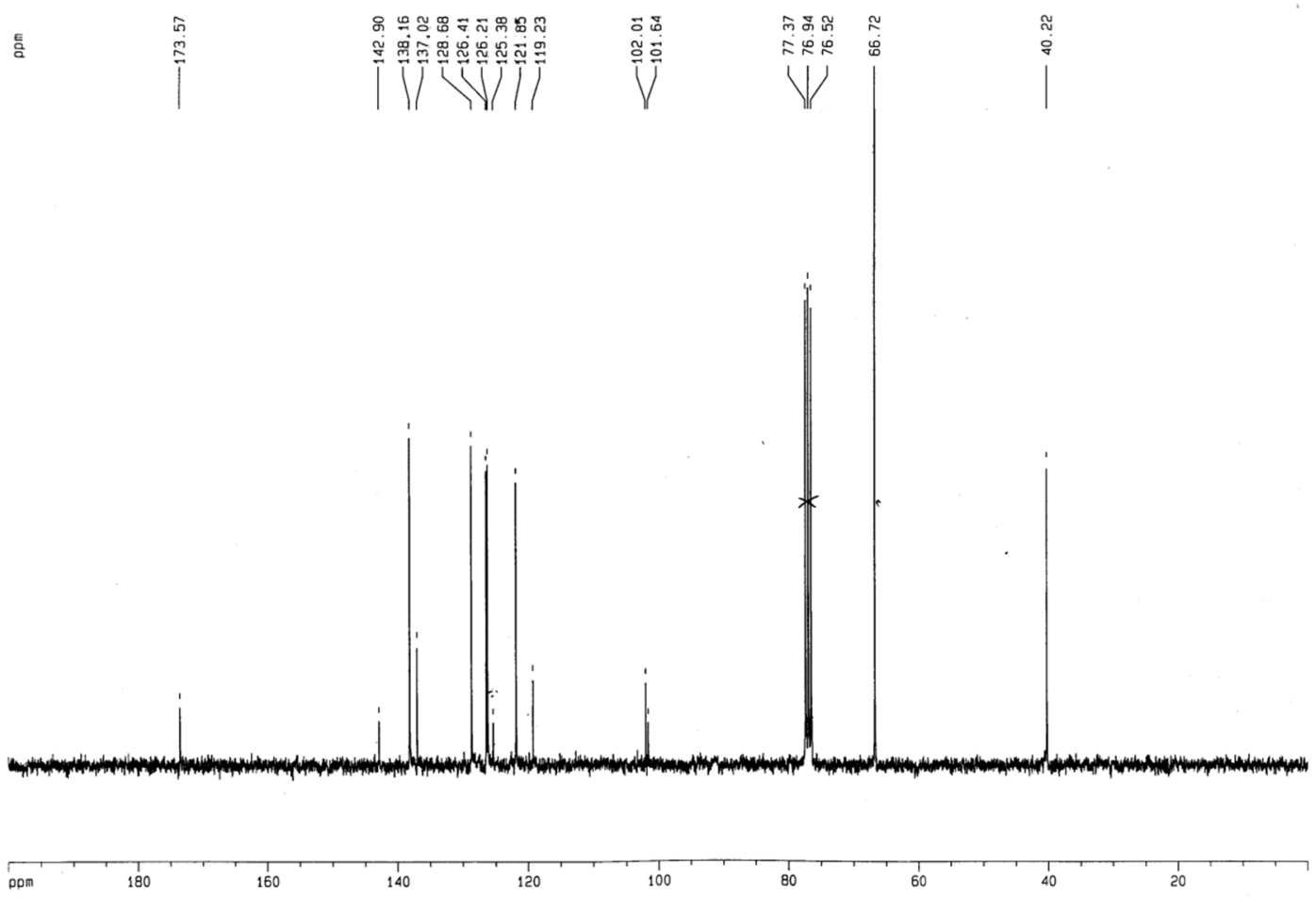
Compound 17

${ }^{1} \mathrm{H}$ NMR $\left(200 \mathrm{MHz}, \mathrm{CDCl}_{3}\right)$

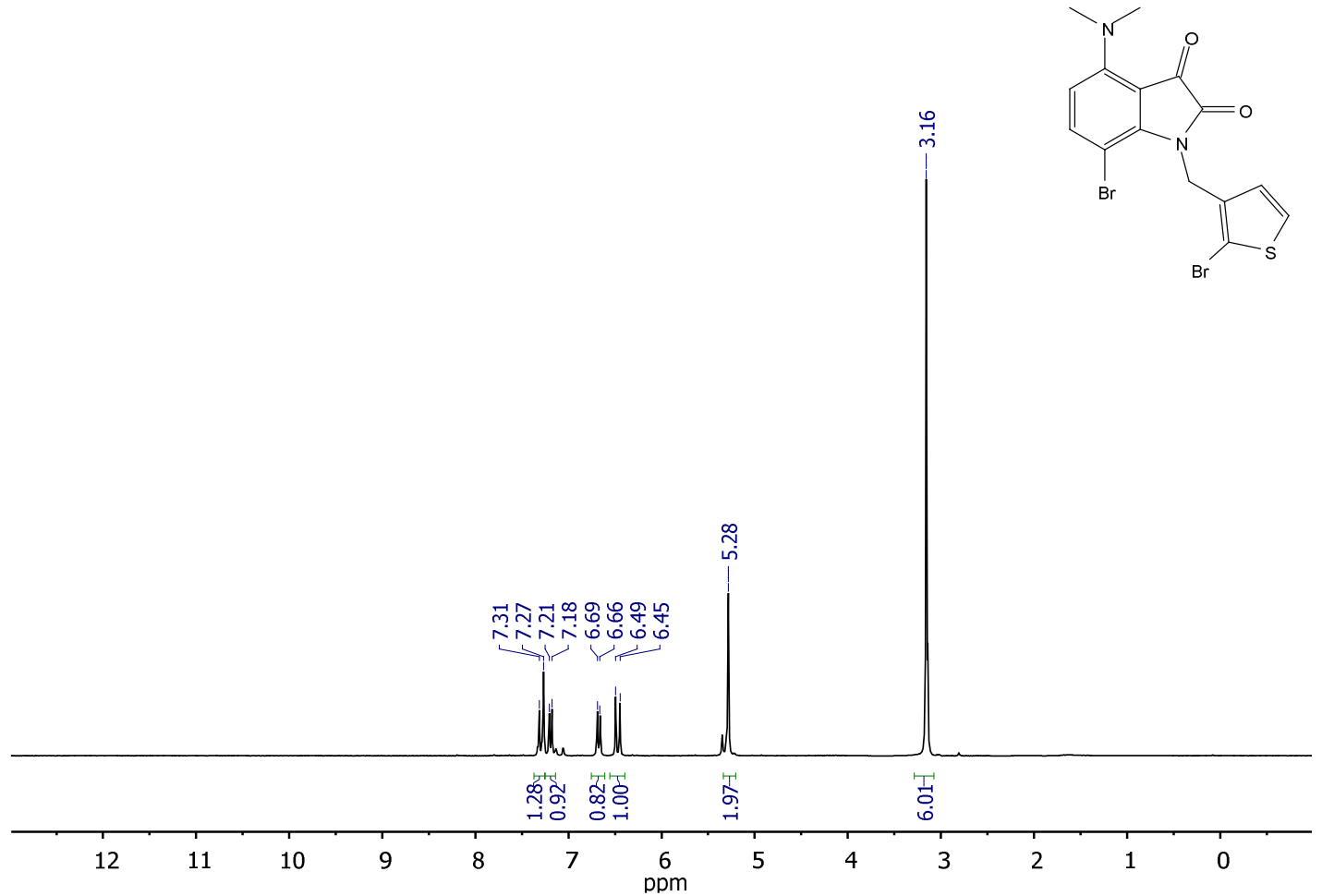

${ }^{13} \mathrm{C}$ NMR $\left(75 \mathrm{MHz}, \mathrm{CDCl}_{3}\right.$ )

言

|

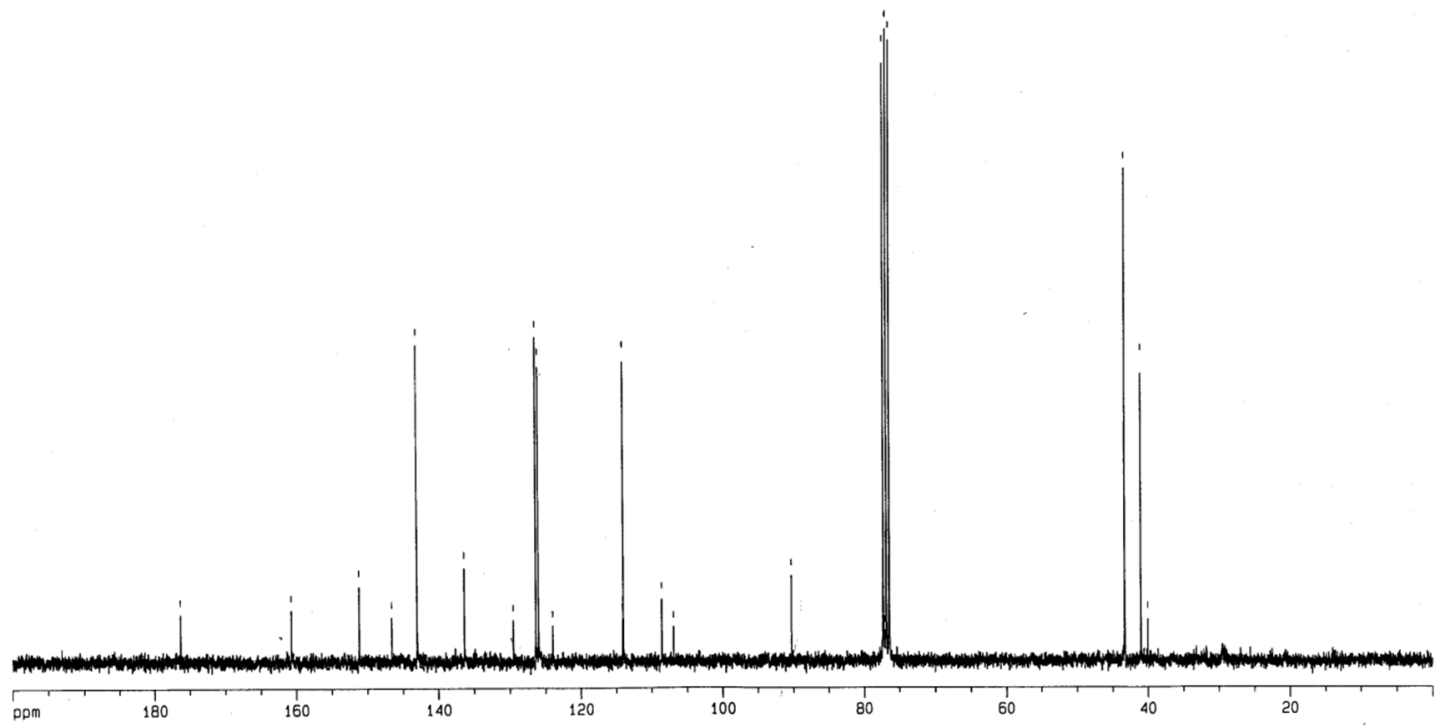




\section{ESI-MS}

NittiFIAO4 \#9 RT: 0.09 AV: 1 NL: $1.78 E 3$
T: ITMS + c ESI Full ms [100.00-2000.00]

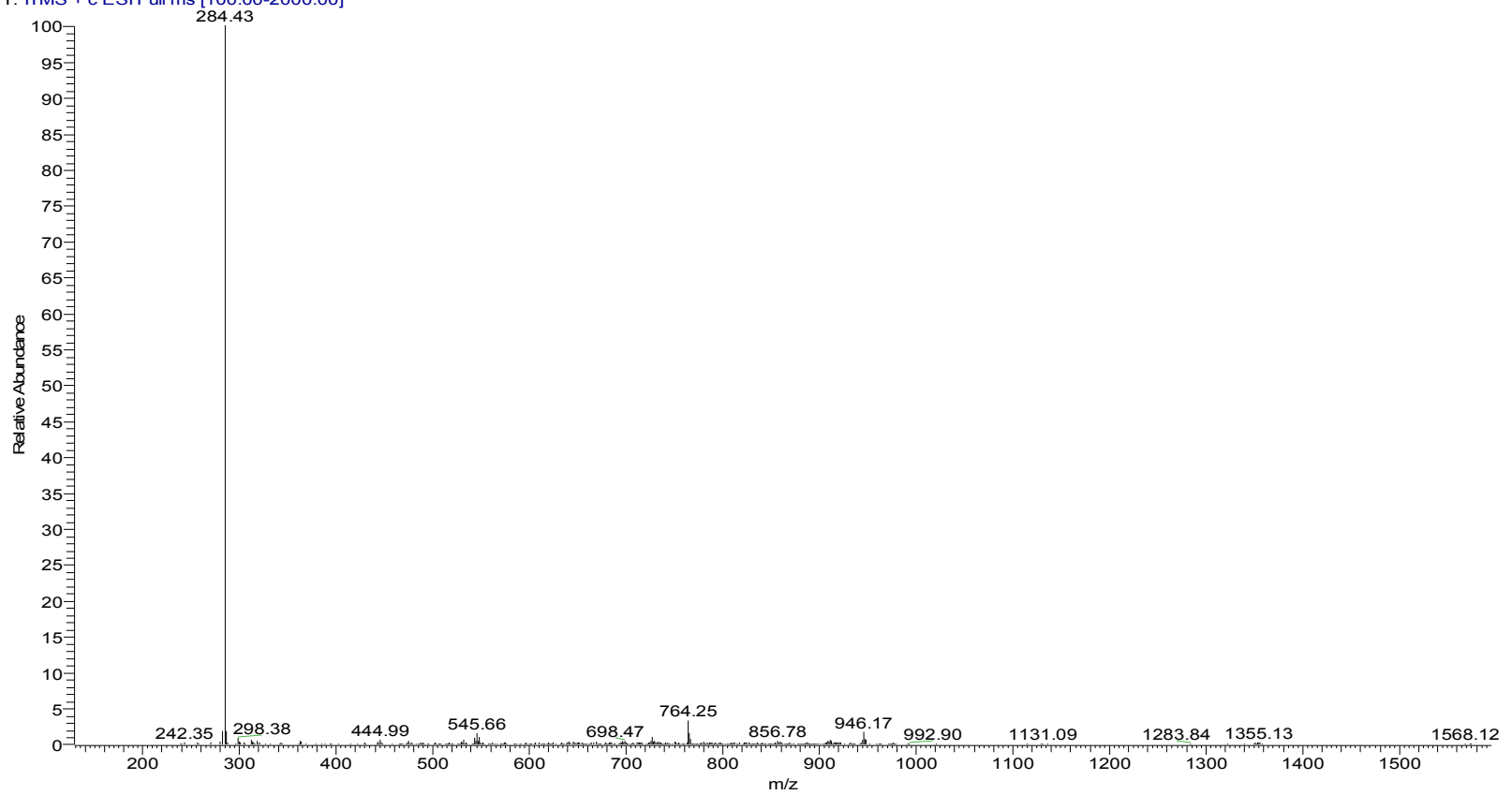




\section{Compound 19}

${ }^{1} \mathrm{H}$ NMR (200 MHz, $d_{6}$-DMSO)
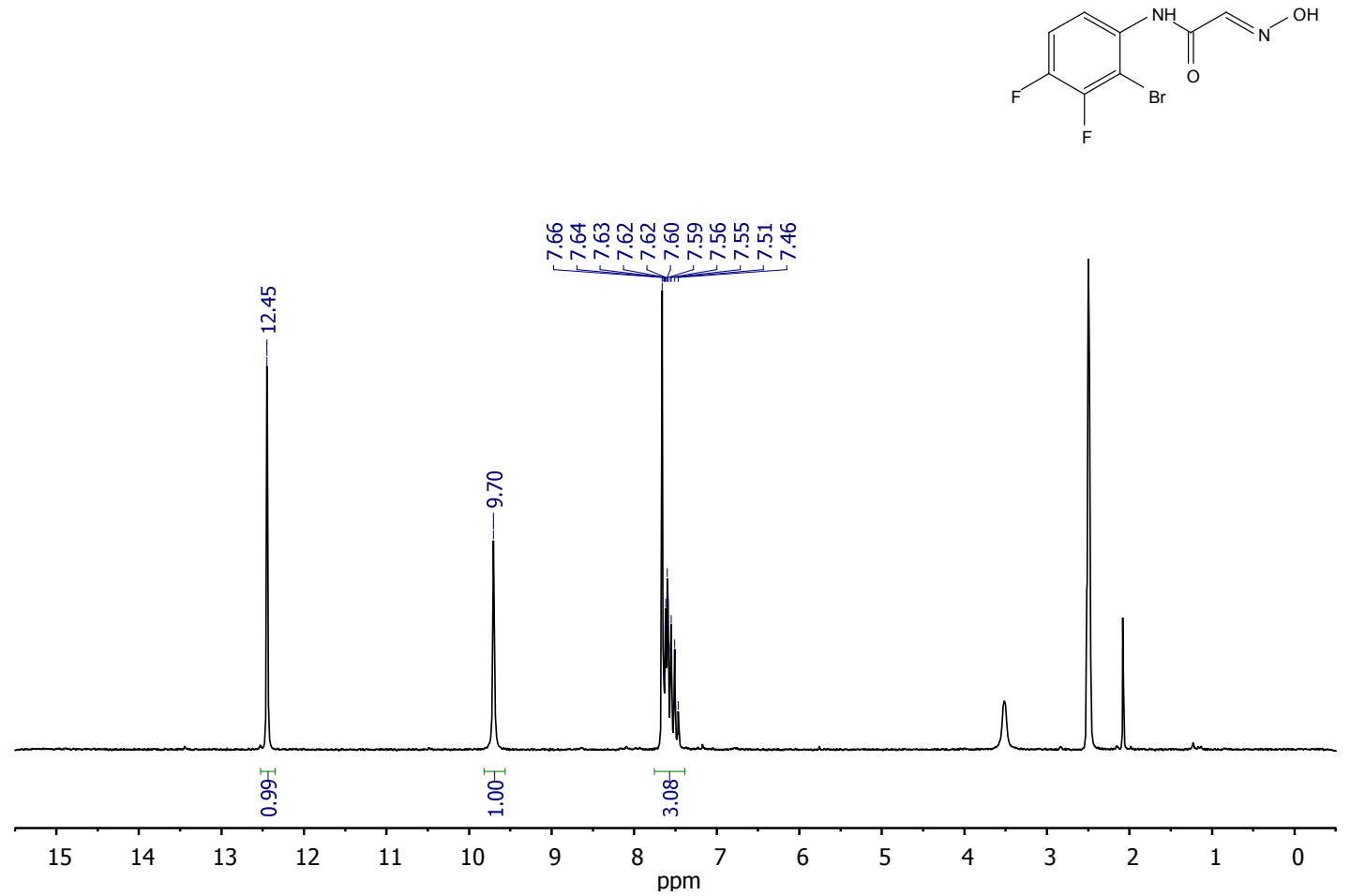

${ }^{13} \mathrm{C}$ NMR (75 MHz, $d_{6}$-DMSO)

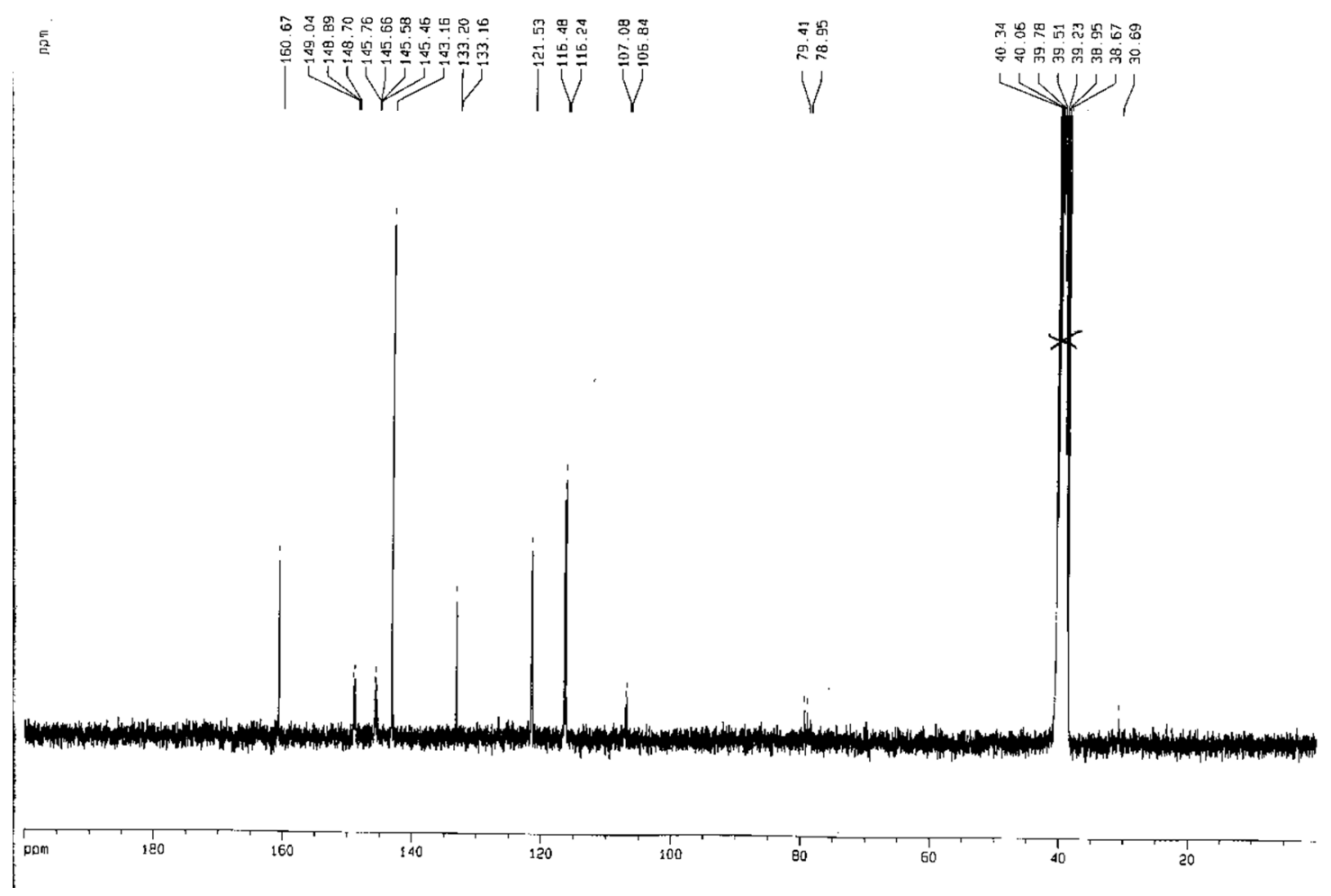


${ }^{1} \mathrm{H}$ NMR (200 MHz, $d_{6}$-DMSO)

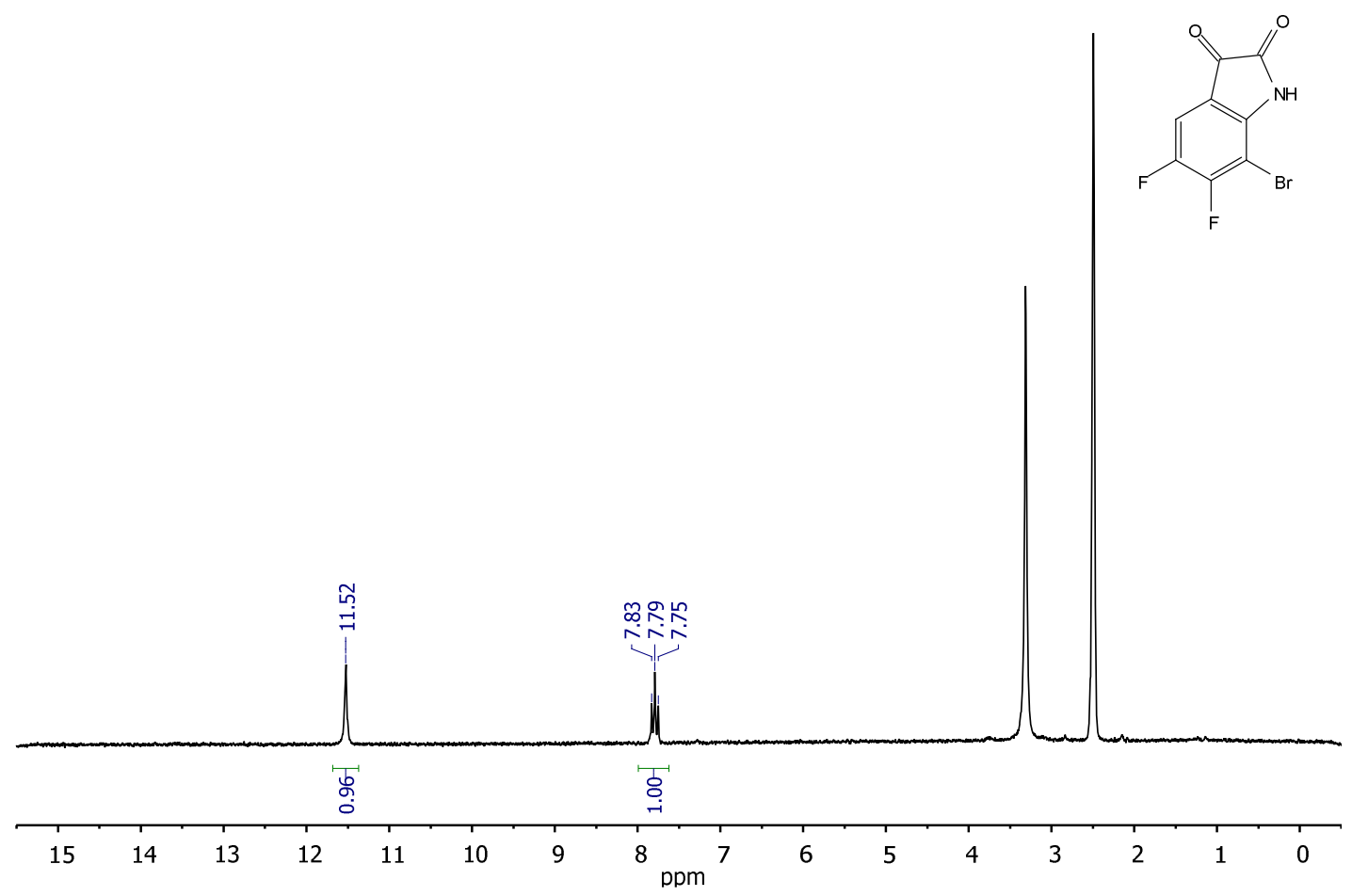

${ }^{13} \mathrm{C}$ NMR (75 MHz, $d_{6}$-DMSO)

言

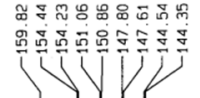

至

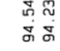

V

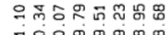

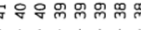

W

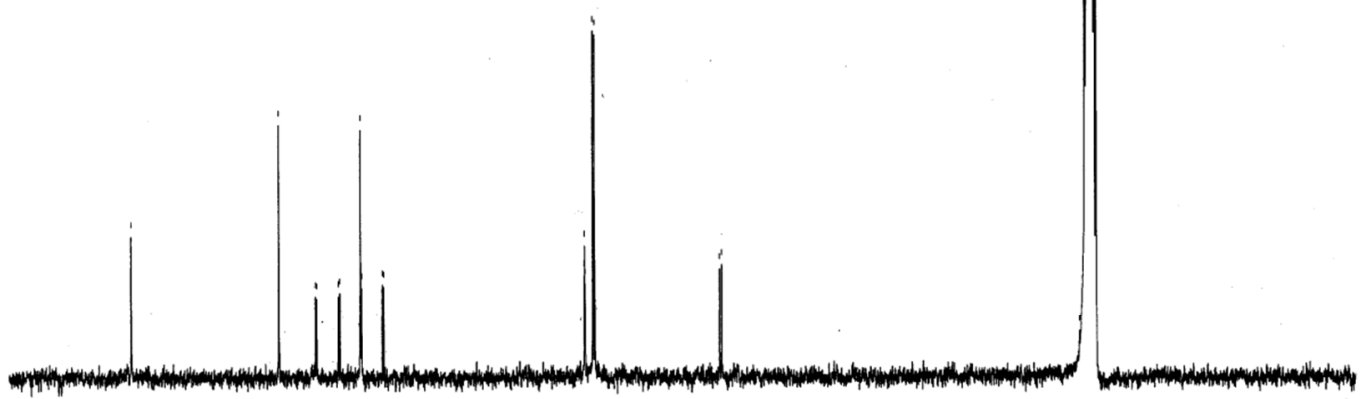

ppm

${ }_{180}^{1}$

160

140

120

100

80

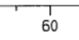

40 


\section{GCMS}

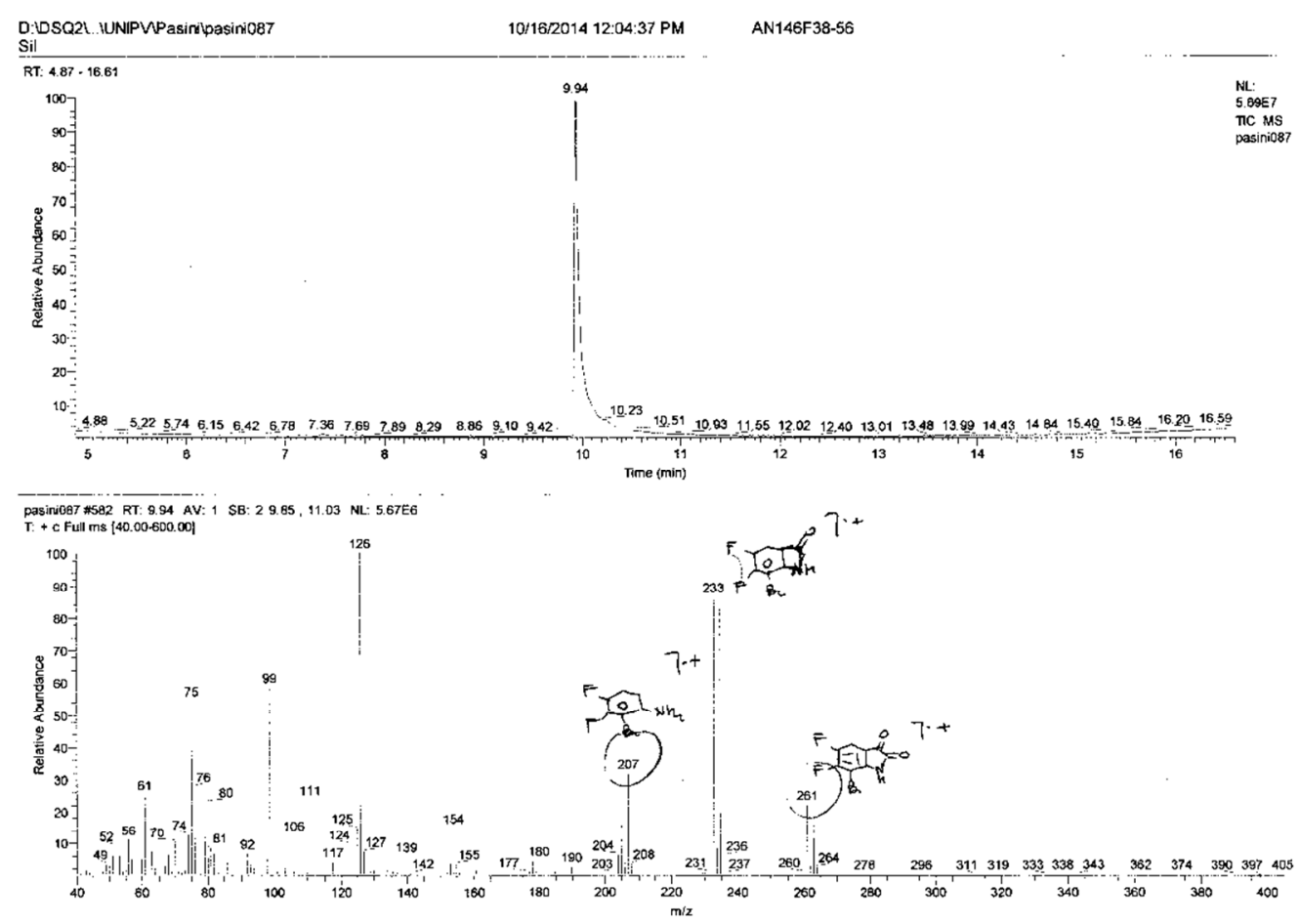




\section{Compound 20}

${ }^{1} \mathrm{H}$ NMR $\left(200 \mathrm{MHz}, \mathrm{CDCl}_{3}\right)$
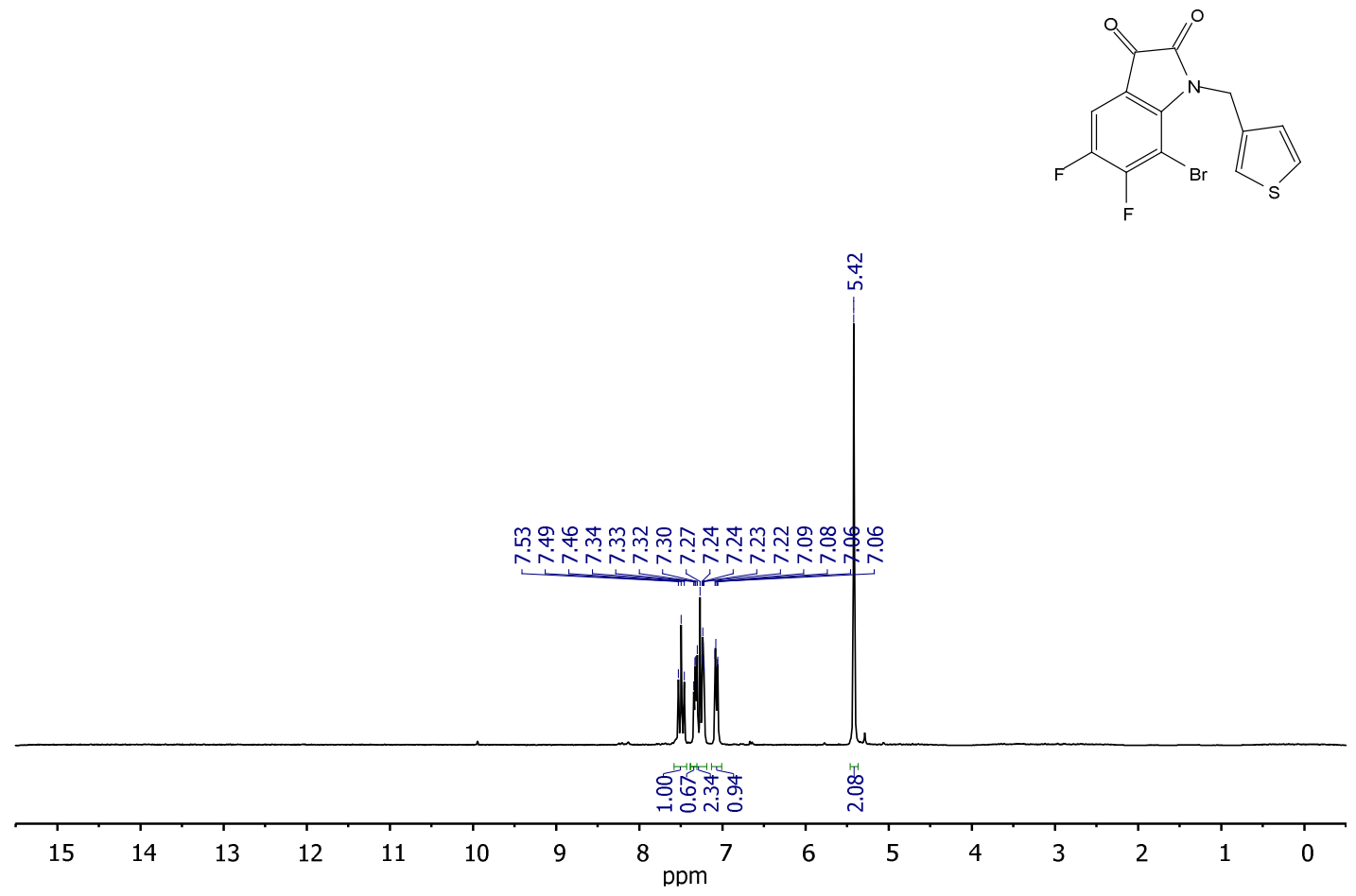

${ }^{13} \mathrm{C}$ NMR $\left(75 \mathrm{MHz}, \mathrm{CDCl}_{3}\right)$

g
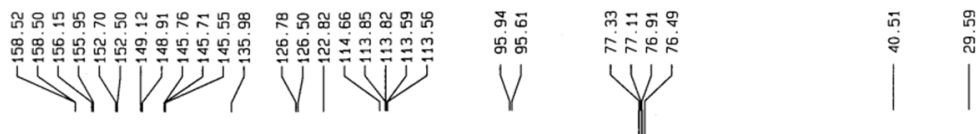

ppm

180

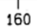

140

120

100

1
80

60 
Compound 21

${ }^{1} \mathrm{H}$ NMR (200 MHz, $\left.\mathrm{CDCl}_{3}\right)$

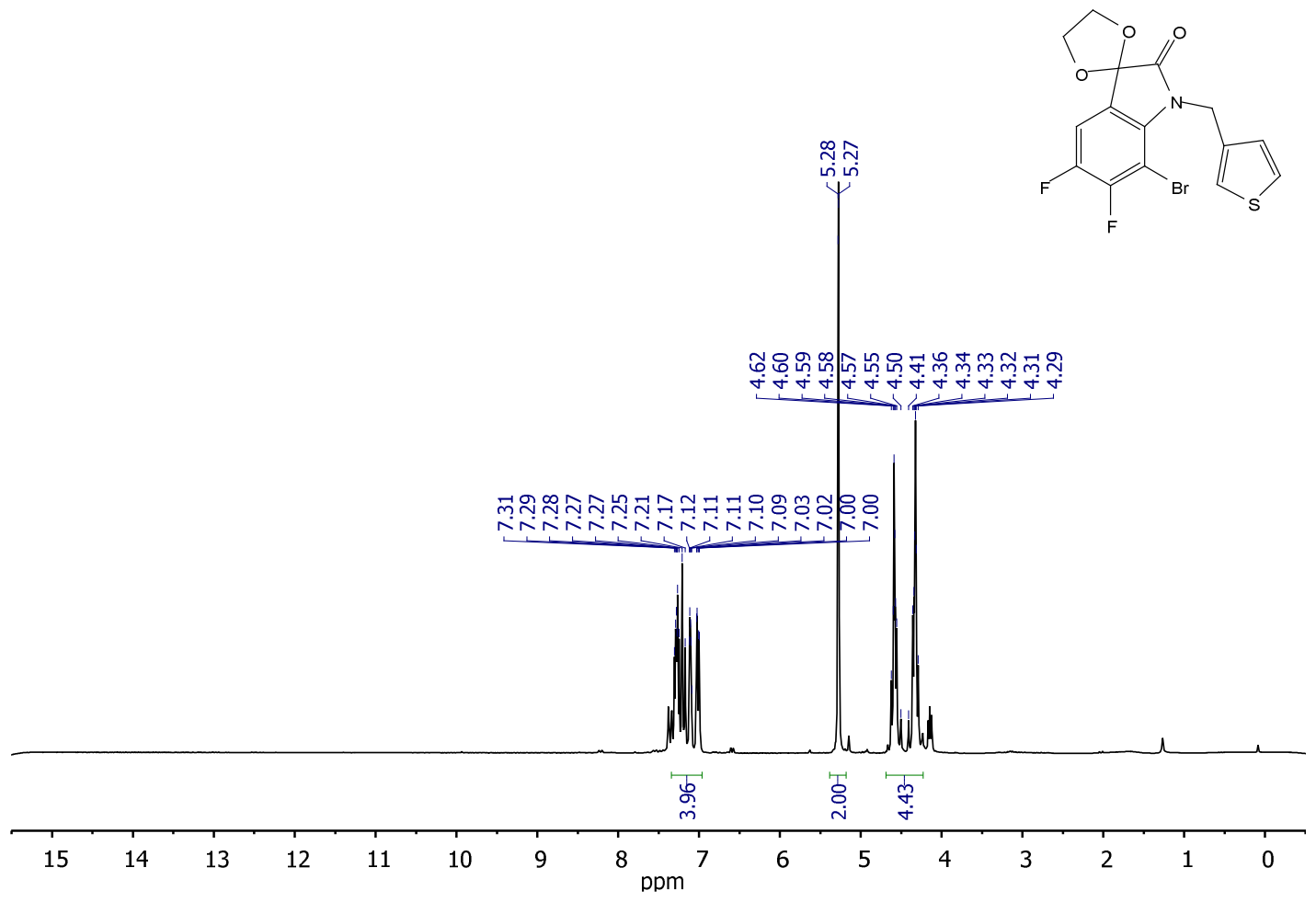

${ }^{13} \mathrm{C}$ NMR $\left(75 \mathrm{MHz}, \mathrm{CDCl}_{3}\right)$
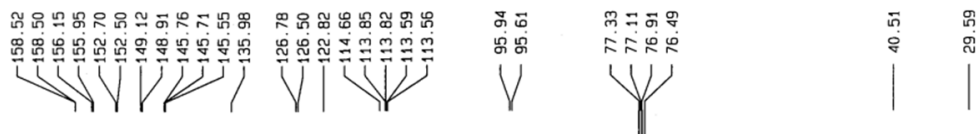

ppm

180

160

140

120

${ }_{100}^{1}$

1
80

60 
Compound 22

${ }^{1} \mathrm{H}$ NMR (200 MHz, $\mathrm{CDCl}_{3}$ )
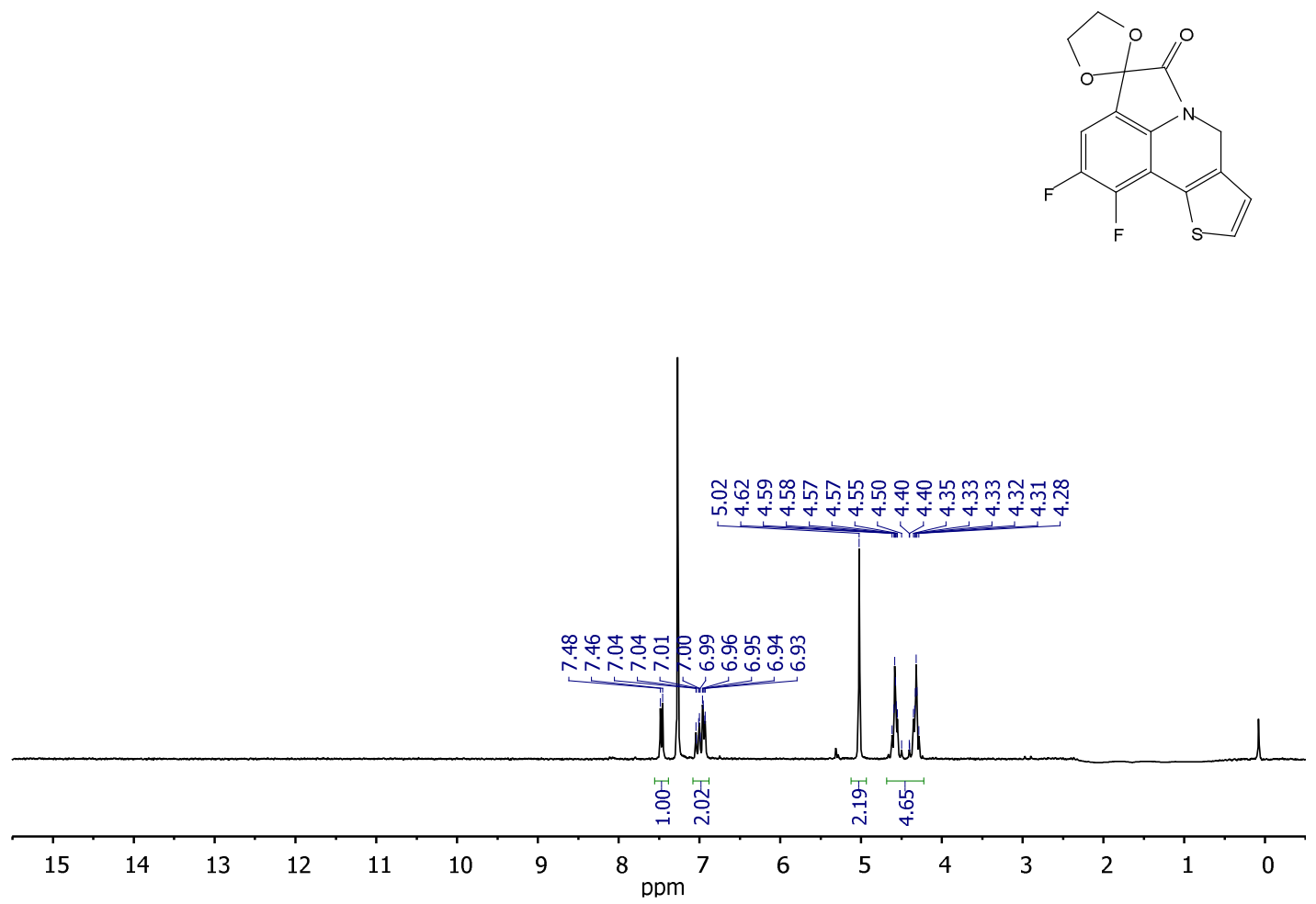

${ }^{13} \mathrm{C}$ NMR $\left(75 \mathrm{MHz}, \mathrm{CDCl}_{3}\right.$ )
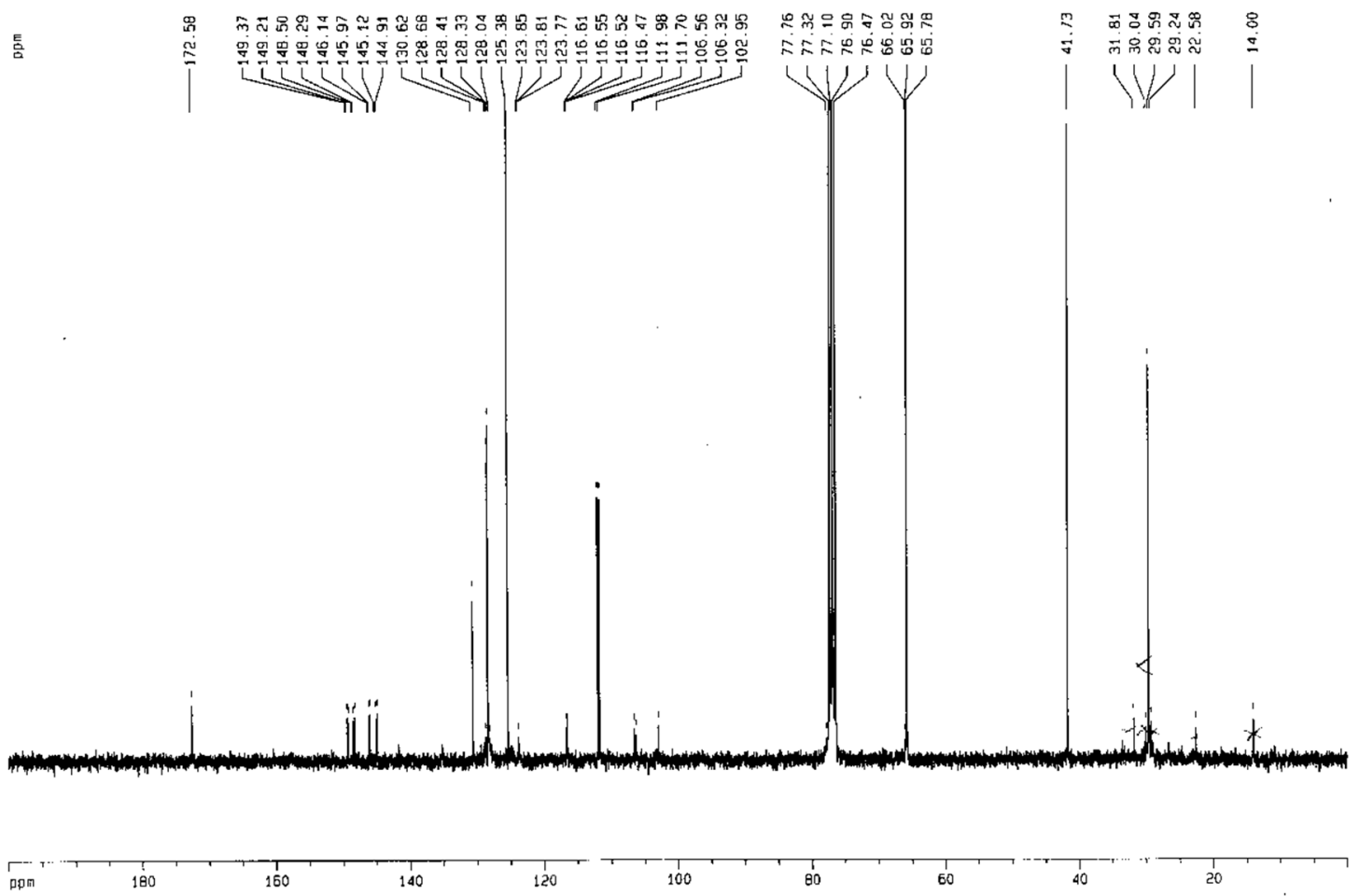


\section{Compound 23}

${ }^{1} \mathrm{H}$ NMR (200 MHz, $\left.\mathrm{CDCl}_{3}\right)$
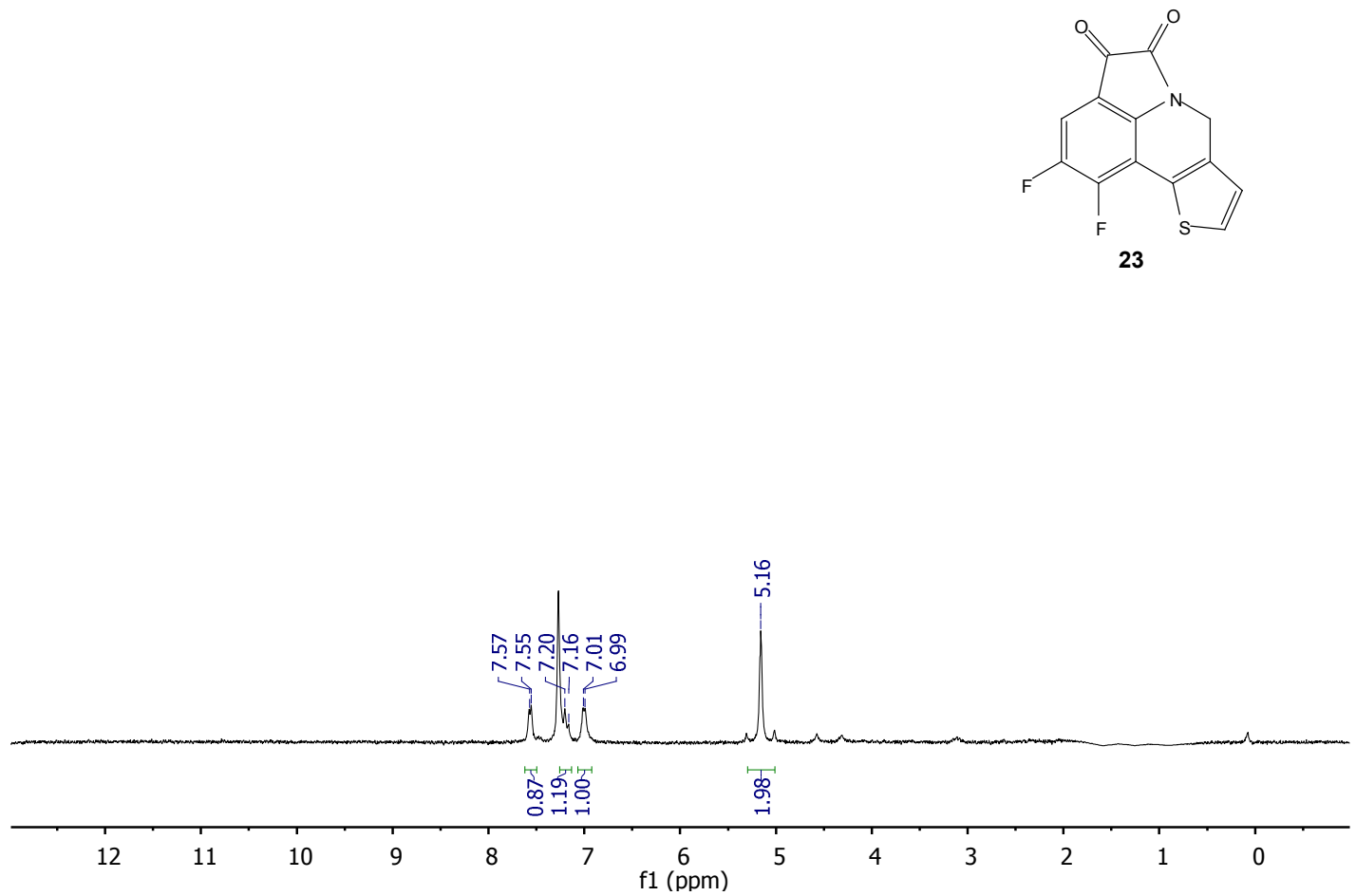

${ }^{13} \mathrm{C}$ NMR $\left(75 \mathrm{MHz}, \mathrm{CDCl}_{3}\right)$

悘
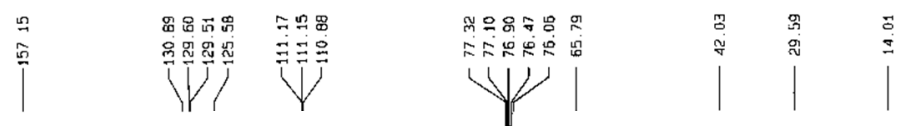

(n)

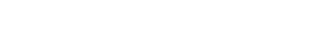



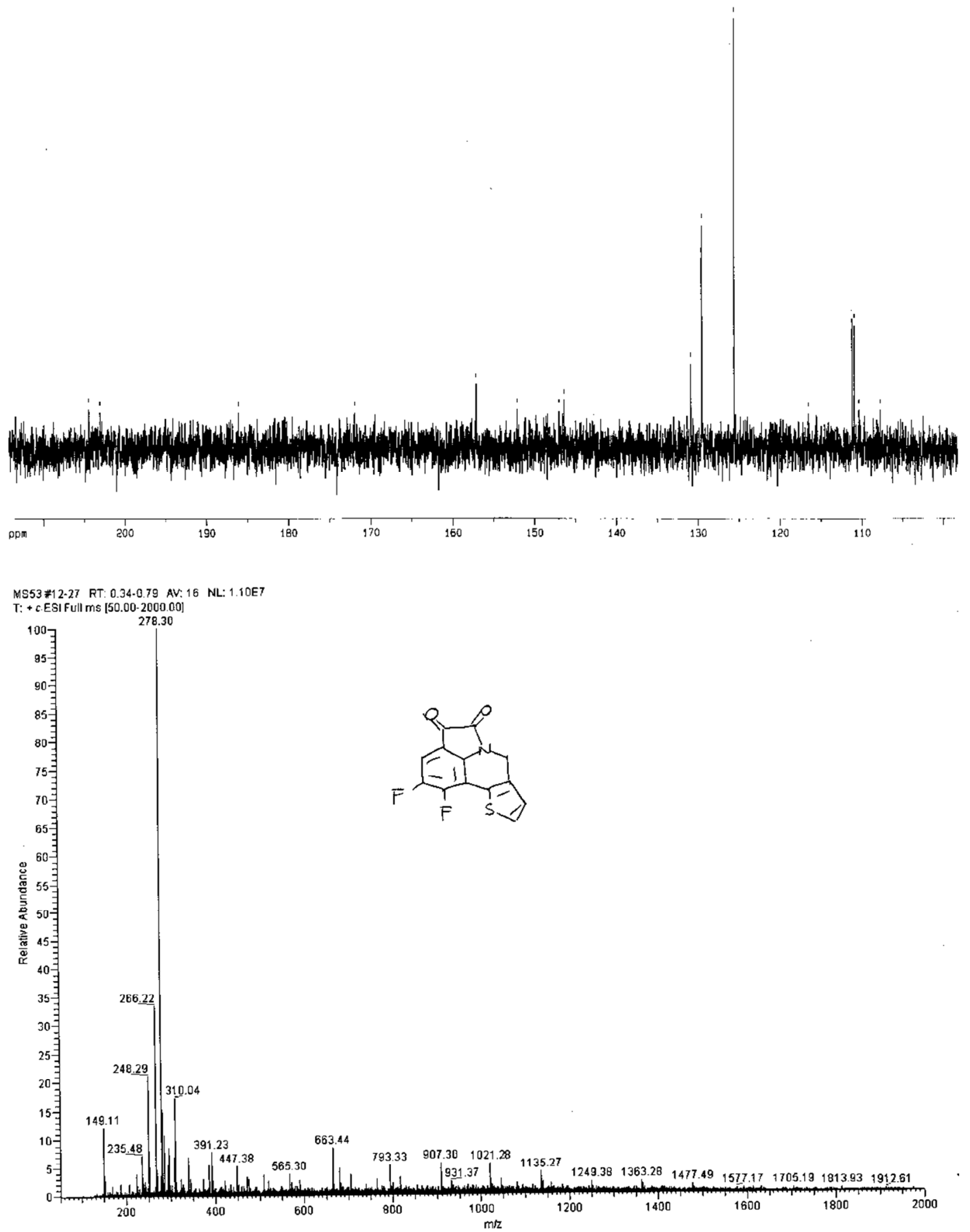